\title{
Nonlinear Stabilization Under Sampled and Delayed Measurements, and With Inputs Subject to Delay and Zero-Order Hold
}

\author{
Iasson Karafyllis and Miroslav Krstic, Fellow, IEEE
}

\begin{abstract}
Sampling arises simultaneously with input and output delays in networked control systems. When the delay is left uncompensated, the sampling period is generally required to be sufficiently small, the delay sufficiently short, and, for nonlinear systems, only semiglobal practical stability is generally achieved. For example, global stabilization of strict-feedforward systems under sampled measurements, sampled-data stabilization of the nonholonomic unicycle with arbitrarily sparse sampling, and sampled-data stabilization of LTI systems over networks with long delays, are open problems. In this paper, we present two general results that address these example problems as special cases. First, we present global asymptotic stabilizers for forward complete systems under arbitrarily long input and output delays, with arbitrarily long sampling periods, and with continuous application of the control input. Second, we consider systems with sampled measurements and with control applied through a zero-order hold, under the assumption that the system is stabilizable under sampled-data feedback for some sampling period, and then construct sampled-data feedback laws that achieve global asymptotic stabilization under arbitrarily long input and measurement delays. All the results employ "nominal" feedback laws designed for the continuous-time systems in the absence of delays, combined with "predictor-based" compensation of delays and the effect of sampling.
\end{abstract}

Index Terms-Feedback stabilization, nonlinear control, sampled-data systems, time-delay systems.

\section{INTRODUCTION}

O AMPLING arises simultaneously with input and output delays in many control problems, most notably in control over networks. In the absence of delays, in sampled-data control of nonlinear systems semiglobal practical stability is generally guaranteed [9], [33]-[35], with the desired region of attraction achieved by sufficiently fast sampling. Alternatively, global results are achieved under restrictive conditions on the structure of the system [8], [11], [15], [16], [18], [37]. On the other hand, in purely continuous-time nonlinear control, input delays of arbitrary length can be compensated [19], [23], [24] but no sam-

Manuscript received December 09, 2010; revised May 14, 2011; accepted September 12, 2011. Date of publication October 03, 2011; date of current version April 19, 2012. Recommended by Associate Editor P. Pepe.

I. Karafyllis is with the Department of Environmental Engineering, Technical University of Crete, 73100, Chania, Greece (e-mail: ikarafyl@enveng.tuc.gr).

M. Krstic is with the Department of Mechanical and Aerospace Engineering, University of California at San Diego, La Jolla, CA 92093-0411 USA (e-mail: krstic@ucsd.edu).

Color versions of one or more of the figures in this paper are available online at http://ieeexplore.ieee.org.

Digital Object Identifier 10.1109/TAC.2011.2170451 pled-data extensions of such results are available. Simultaneous consideration to sampling and delays (either physical or sampling-induced) is given in the literature on control of linear and nonlinear systems over networks [6], [7], [10], [32], [36], [37], [40], [41], [43], but almost all available results rely on delay-dependent conditions for the existence of stabilizing feedback. Exceptions are the papers [2], [27], where prediction-based control methodologies are employed.

Despite the remarkable accomplishments in the fields of sampled-data, networked, and nonlinear delay systems, the following example problems remain open: global stabilization of strict-feedforward systems under sampled measurements and continuous control, sampled-data stabilization of the nonholonomic unicycle under arbitrarily sparse sampling, and sampled-data stabilization of LTI systems over networks with long delays.

In this paper, we introduce two frameworks for solving such problems in two distinctly different categories:

1) We present global asymptotic stabilizers for forward complete systems (systems whose solutions exist for all time, irrespective of initial condition and input) under arbitrarily long input and output delays, with arbitrarily long sampling periods, and with continuous application of the control input.

2) We consider systems with sampled measurements and with control applied through a zero-order hold, under the assumption that the system is stabilizable under sampleddata feedback for some sampling period, and then construct sampled-data feedback laws that achieve global asymptotic stabilization under arbitrarily long input and measurement delays.

In both frameworks we employ "nominal" feedback laws designed in the absence of delays, combined with "predictor-based" compensation of delays.

1) Problem Statement: As in [19], [23]-[25], [28]-[30], [42], [44], we consider systems with input delay

$$
\dot{x}(t)=f(x(t), u(t-\tau))
$$

where $x(t)=\left(x_{1}(t), \ldots, x_{n}(t)\right)^{\prime} \in \Re^{n}, u(t) \in \Re^{m}, f:$ $\Re^{n} \times \Re^{m} \rightarrow \Re^{n}$ is a locally Lipschitz mapping with $f(0,0)=0$ and $\tau \geq 0$ is a constant. In [9], [23]-[25], and [44], the feedback design problem for system (1) is addressed by assuming a feedback stabilizer $u=k(x)$ for system (1) with no delay, i.e., (1) with $\tau=0$, or

$$
\dot{x}(t)=f(x(t), u(t))
$$


and applying a delay compensator (predictor) methodology based on the knowledge of the delay. In this paper, we incorporate also a consideration of measurement delay, namely, we address the problem of stabilization of (1) with output

$$
y(t)=x(t-r) \in \Re^{n}
$$

where $r \geq 0$ is a constant, i.e., we consider delayed measurements. The motivation for a simultaneous consideration of input and measurement delays is that in many chemical process control problems the measurement delay of concentrations of chemical species can be large.

We also assume that the output is available at discrete time instants $\tau_{i}$ (the sampling times) with $\tau_{i+1}-\tau_{i}=T>0$, where $T>0$ is the sampling period. Very few papers have studied this problem (an exception is [12] where input and measurement delays are considered for linear systems but the measurement is not sampled).

The problem of stabilization of (1) with output given by (3) is intimately related to the stabilization of system (1) alone. To see this, notice that the output $y(t)$ of (1), (3) satisfies the following system of differential equations for all $t \geq r$ :

$$
\dot{y}(t)=f(y(t), u(t-r-\tau)) .
$$

Consider the comparison between two problems described by the same differential equations: the problem of stabilization of (1) with input delay $r>0$ and no measurement delay (i.e., $\dot{x}(t)=f(x(t), u(t-r))$ for all $t \geq 0)$ and the problem of stabilization of (1), (3) with no input delay and measurement delay $r>0$ (i.e., $\dot{y}(t)=f(y(t), u(t-r))$ for all $t \geq r$ ). The two problems are not identical: in the first stabilization problem the applied input values for $t \in[0, r]$ are given (as initial conditions), while in the second stabilization problem the applied input values for $t \in[0, r]$ must be computed based on an arbitrary initial condition $x(\theta)=x_{0}(\theta), \theta \in[-r, 0]$ (irrespective of the current value of the state). Therefore, serious technical issues concerning the existence of the solution for $t \in[0, r]$ arise for the second stabilization problem (see Remark 2.2(b) below).

2) Results of the Paper: We establish two general results:

1) A solution for the stabilization of (1) with output given by (3) under the assumption that system (2) is globally stabilizable and forward complete and the input can be continuously adjusted (Theorem 2.1). The proposed dynamic sampled-data controller uses values of the output (3) at the discrete time instants $\tau_{i}=t_{0}+i T, i \in Z^{+}$, where $T>0$ is the sampling period and $t_{0} \geq 0$ is the initial time. This justifies the term "sampled-data." No restrictions for the values of the delays $r, \tau \geq 0$ or the sampling period $T>0$ are imposed. In general, we show that there is no need for continuous measurements for global asymptotic stabilization of any stabilizable forward complete system with arbitrary input and output delays.

2) A solution for the stabilization of (1) with output given by (3) under the assumption that system (2) is globally stabilizable and forward complete and the control action is implemented with zero order hold (Theorem 3.2). Again, the proposed sampled-data controller uses values of the output (3) at the discrete time instants $\tau_{i}=t_{0}+i T, i \in Z^{+}$, where
$T>0$ is the sampling period and $t_{0} \geq 0$ is the initial time. In this case, we can solve the stabilization problem for systems with both delayed inputs and measurements.

Our delay compensation methodology guarantees that any controller (continuous or sampled-data) designed for the delayfree case can be used for the regulation of the delayed system with input/measurement delays and sampled measurements. For example, all sampled-data feedback designs proposed in [8], [9], [15], [18], [33], [34], [35], and [37] which guarantee global stabilization can be exploited for the stabilization of a delayed system with input/measurement delays, sampled measurements and input applied with zero order hold.

The results are applied to the following:

- The linear time invariant (LTI) case, where $f(x, u)=$ $A x+B u, A \in \Re^{n \times n}, B \in \Re^{n \times m}$. This case has been recently studied extensively in the context of linear networked control systems, where various delays arise. Delay-dependent and/or sampling period-dependent sufficient conditions for the stabilization of networked control systems have been proposed in the literature [6], [7], [10], [32], [36], [37], [40], [41], [43]. Here, we propose a linear delay compensator that guarantees exponential stability of the closed-loop system with no restrictions for the delays (Corollary 3.4). The compensator is designed based on the knowledge of linear feedback stabilizer for the delay-free case.

- Strict-feedforward systems [22], [24], [39], which are studied in Examples in 2.4 and 3.8.

- The stabilization of the nonholonomic integrator

$$
\dot{x}_{1}=u_{1} \quad, \quad \dot{x}_{2}=x_{1} u_{2} \quad, \quad \dot{x}_{3}=u_{2}
$$

with both delayed inputs and measurements. The problem was recently studied in [21] in the presence of delays and in [8], [35] in the presence of sampling. Here, our proposed dynamic sampled-data controller is applied with no restrictions for the value of the delays or the size of the sampling period. Two solutions are presented: one that guarantees asymptotic stability (Corollary 4.1) and one that guarantees finite-time stability (Proposition 4.2).

Since the proposed delay compensation feedback design methodology is based on the prediction of the state, explicit formulae for the predictor mapping will give explicit formulae for the feedback stabilizer. Explicit formulae for the predictor mapping can be provided for a limited class of nonlinear systems (see Remark 2.2(d) below). However, recent results have shown that the implementation of the predictor mapping can be done without the knowledge of an explicit formula (e.g., by solving a system of first order partial differential equations as in [24]). Moreover, recent results have shown that the predictor mapping can be approximated by numerical schemes so that the implementation of the controller can be made without the knowledge of an explicit formula (see [19]).

3) Organization of the Paper: In Section II, the main results concerning the case of the continuously adjusted input are stated and many comments and explanations are provided. In Section III, the main results concerning the case of input applied with zero order hold are provided. Special results are provided for the case of linear autonomous systems and for the case 
of nonlinear systems which are diffeomorphically equivalent to a chain of integrators. Section IV is devoted to the application of the obtained results to the stabilization of a three-wheeled vehicle with two independent rear motorized wheels (the nonholonomic integrator). Finally, in Section $\mathrm{V}$ we present the concluding remarks of the present work. The Appendix contains the proofs of certain results.

Notation: Throughout the paper we adopt the following notation:

- For a vector $x \in \Re^{n}$ we denote by $|x|$ its usual Euclidean norm, by $x^{\prime}$ its transpose. For a real matrix $A \in \Re^{n \times m}, A^{\prime} \in \Re^{m \times n}$ denotes its transpose and $|A|:=\sup \left\{|A x| ; x \in \Re^{n},|x|=1\right\}$ is its induced norm. $I \in \Re^{n \times n}$ denotes the identity matrix.

- $\Re^{+}$denotes the set of non-negative real numbers. $Z^{+}$denotes the set of non-negative integers. For every $t \geq 0,[t]$ denotes the integer part of $t \geq 0$, i.e., the largest integer being less or equal to $t \geq 0$.

- For the definition of the class of functions $K L$, see [20].

- By $C^{j}(A)\left(C^{j}(A ; \Omega)\right)$, where $A \subseteq \Re^{n}\left(\Omega \subseteq \Re^{m}\right), j \geq 0$ is a non-negative integer, we denote the class of functions (taking values in $\Omega \subseteq \Re^{m}$ ) that have continuous derivatives of order $j$ on $A \subseteq \Re^{n}$.

- Let $x:[a-r, b) \rightarrow \Re^{n}$ with $b>a \geq 0$ and $r \geq 0$. By $T_{r}(t) x$ we denote the "history" of $x$ from $t-r$ to $t$, i.e., $\left(T_{r}(t) x\right)(\theta):=x(t+\theta) ; \theta \in[-r, 0]$, for $t \in[a, b)$. By $T_{r}(t) x$ we denote the "open history" of $x$ from $t-r$ to $t$, i.e., $\left(\widetilde{T}_{r}(t) x\right)(\theta):=x(t+\theta) \theta \in[-r, 0)$, for $t \in[a, b)$.

- Let $I \subseteq \Re^{+}:=[0,+\infty)$ be an interval. By $L^{\infty}(I ; U)$ $\left(L_{l o c}^{\infty}(I ; U)\right)$ we denote the space of measurable and (locally) bounded functions $u(\cdot)$ defined on $I$ and taking values in $U \subseteq \Re^{m}$. Notice that we do not identify functions in $L^{\infty}(\bar{I} ; U)$ which differ on a measure zero set. For $x \in L^{\infty}\left([-r, 0] ; \Re^{n}\right)$ or $x \in L^{\infty}\left([-r, 0) ; \Re^{n}\right)$ we define $\|x\|_{r}:=\sup _{\theta \in[-r, 0]}|x(\theta)|$ or $\|x\|_{r}:=\sup _{\theta \in[-r, 0)}|x(\theta)|$. Notice that $\sup _{\theta \in[-r, 0]}|x(\theta)|$ is not the essential supremum but the actual supremum and that is why the quantities $\sup _{\theta \in[-r, 0]}|x(\theta)|$ and $\sup _{\theta \in[-r, 0)}|x(\theta)|$ do not coincide in general. We will also use the notation $M_{U}$ for the space of measurable and locally bounded functions $u: \Re^{+} \rightarrow U$.

- We say that a system of the form (2) is forward complete if for every $x_{0} \in \Re^{n}, u \in M_{U}$ the solution $x(t)$ of (2) with initial condition $x(0)=x_{0} \in \Re^{n}$ corresponding to input $u \in M_{U}$ exists for all $t \geq 0$.

Throughout the paper we adopt the convention $L^{\infty}\left([-r, 0] ; \Re^{n}\right)=\Re^{n}$ and $C^{0}\left([-r, 0] ; \Re^{n}\right)=\Re^{n}$ for $r=0$. Finally, for reader's convenience, we mention the following fact, which is a direct consequence of Lemma 2.2 in [1] and Lemma 3.2 in [14]. The fact is used extensively throughout the paper.

4) Fact: Suppose that system (2) is forward complete. Then for every $x_{0} \in \Re^{n}, u \in L_{l o c}^{\infty}\left([-\tau,+\infty) ; \Re^{m}\right)$ the solution $x(t)$ of (1) with initial condition $x(0)=x_{0} \in \Re^{n}$ corresponding to input $u \in L_{l o c}^{\infty}\left([-\tau,+\infty) ; \Re^{m}\right)$ exists for all $t \geq 0$. Moreover, for every $T>0$ there exists a function $a \in K_{\infty}$ such that for every $x_{0} \in \Re^{n}, u \in L_{l o c}^{\infty}\left([-\tau,+\infty) ; \Re^{m}\right)$ the solu- tion $x(t)$ of (1) with initial condition $x(0)=x_{0} \in \Re^{n}$ corresponding to input $u \in L_{l o c}^{\infty}\left([-\tau,+\infty) ; \Re^{m}\right)$ satisfies $|x(t)| \leq$ $a\left(\left|x_{0}\right|+\sup _{-\tau \leq s<t-\tau}|u(s)|\right)$, for all $t \in(0, T]$.

\section{Dynamic SAmpled-Data FeEdback For CONTINUOUSLY ADJUSTED INPUT}

We start by presenting the assumptions for system (2). Our first assumption concerning system (2) is forward completeness.

Hypothesis (H1): System (2) is forward complete.

Assumption (H1) guarantees that system (1) is forward complete as well: for every $x_{0} \in \Re^{n}, u \in L_{l o c}^{\infty}\left([-\tau,+\infty) ; \Re^{m}\right)$ the solution $x(t)$ of (1) with initial condition $x(0)=x_{0} \in \Re^{n}$ corresponding to input $u \in L_{l o c}^{\infty}\left([-\tau,+\infty) ; \Re^{m}\right)$ exists for all $t \geq 0$. Therefore, we are in a position to define the "predictor" mapping $\Phi: \Re^{n} \times L^{\infty}\left([-r-\tau, 0) ; \Re^{m}\right) \rightarrow \Re^{n}$ for all $r, \tau \geq 0$ with $r+\tau>0$ in the following way:

"For every $x_{0} \in \Re^{n}, u \in L^{\infty}\left([-r-\tau, 0) ; \Re^{m}\right)$ the solution $x(t)$ of (1) with initial condition $x(-r)=x_{0}$ corresponding to input $u \in L^{\infty}\left([-r-\tau, 0) ; \Re^{m}\right)$ satisfies $x(\tau)=\Phi\left(x_{0}, u\right)$ "

By virtue of the fact, we can guarantee the existence of $a \in$ $K_{\infty}$ such that

$$
\begin{aligned}
|\Phi(x, u)| & \leq a\left(|x|+\|u\|_{r+\tau}\right), \\
\text { for all }(x, u) & \in \Re^{n} \times L^{\infty}\left([-r-\tau, 0) ; \Re^{m}\right) .
\end{aligned}
$$

Using (5) and the fact that $f: \Re^{n} \times \Re^{m} \rightarrow \Re^{n}$ is a locally Lipschitz mapping, we can guarantee the existence of a nondecreasing function $L: \Re^{+} \rightarrow \Re^{+}$such that

$$
\begin{aligned}
& \quad|\Phi(x, u)-\Phi(y, v)| \\
& \quad \leq L\left(|x|+|y|+\|u\|_{r+\tau}+\|v\|_{r+\tau}\right)\left(|x-y|+\|u-v\|_{r+\tau}\right), \\
& \quad \text { for all } x, y \in \Re^{n}, u, v \in L^{\infty}\left([-r-\tau, 0) ; \Re^{m}\right) .
\end{aligned}
$$

We assume next that (2) is globally stabilizable.

Hypothesis (H2) (Continuously Adjusted Input): There exists $k \in C^{1}\left(\Re^{+} \times \Re^{n} ; \Re^{m}\right), g \in K_{\infty}$ with

$$
|k(t, x)| \leq g(|x|), \text { for all }(t, x) \in \Re^{+} \times \Re^{n}
$$

such that $0 \in \Re^{n}$ is uniformly globally asymptotically stable for system (2) with $u=k(t, x)$, i.e., there exists a function $\sigma \in K L$ such that for every $\left(t_{0}, x_{0}\right) \in \Re^{+} \times \Re^{n}$ the solution $x(t)$ of (2) with $u=k(t, x)$ and initial condition $x\left(t_{0}\right)=x_{0} \in \Re^{n}$ satisfies the following inequality:

$$
|x(t)| \leq \sigma\left(\left|x_{0}\right|, t-t_{0}\right), \forall t \geq t_{0} .
$$

Consider system (1) under hypotheses (H1), (H2) for system (2). Our proposed dynamic sampled-data feedback has states $\left(z(t), T_{r+\tau}(t) u\right) \in \Re^{n} \times L^{\infty}\left([-r-\tau, 0] ; \Re^{m}\right)$ and inputs $y(t) \in \Re^{n}$ and for each $t_{0} \geq 0,\left(z_{0}, u_{0}\right) \in$ $\Re^{n} \times L^{\infty}\left([-r-\tau, 0] ; \Re^{m}\right)$ the states are computed by the interconnection of two subsystems:

1) A sampled-data subsystem (see [14]) with inputs $\left(y(t), T_{r+\tau}(t) u\right) \in \Re^{n} \times L^{\infty}\left([-r-\tau, 0] ; \Re^{m}\right)$

$$
\begin{aligned}
\dot{z}(t) & =f(z(t), u(t)), t \in\left[\tau_{i}, \tau_{i+1}\right), i \in Z^{+} \\
z\left(\tau_{i+1}\right) & =\Phi\left(y\left(\tau_{i+1}\right), \breve{T}_{r+\tau}\left(\tau_{i+1}\right) u\right) \\
z\left(t_{0}\right) & =z_{0} \in \Re^{n}
\end{aligned}
$$


where

$$
\tau_{i}=t_{0}+i T, i \in Z^{+}
$$

are the sampling times and $T>0$ is the sampling period. We stress that the proposed sampled-data dynamic controller uses only values of the output $y(t)=x(t-r) \in \Re^{n}$ at the discrete time instants $\tau_{i}=t_{0}+i T$, where $i \in Z^{+}$.

2) A subsystem described by functional difference equations (see [17]) with inputs $z(t) \in \Re^{n}$

$$
\begin{aligned}
u(t) & =k(t+\tau, z(t)), t>t_{0} \\
T_{r+\tau}\left(t_{0}\right) u & =u_{0} \in L^{\infty}\left([-r-\tau, 0] ; \Re^{m}\right) .
\end{aligned}
$$

It is clear that during the intersampling interval, the controller employs a copy of the system to be controlled. The purpose is to predict the evolution of the states and reminds similar methodologies employed in control systems under communication constraints (see [3], [26]). Our first main result is now stated.

Theorem 2.1: Let $T>0, r, \tau \geq 0$ with $r+\tau>0$ and suppose that hypotheses (H1), (H2) hold for system (2). Then the closedloop system (1), (3), (9), (10) is uniformly globally asymptotically stable, in the sense that there exists a function $\tilde{\sigma} \in K L$ such that for every $t_{0} \geq 0,\left(x_{0}, z_{0}, u_{0}\right) \in C^{0}\left([-r, 0] ; \Re^{n}\right) \times$ $\Re^{n} \times L^{\infty}\left([-r-\tau, 0] ; \Re^{m}\right)$, the solution $(x(t), z(t), u(t)) \in$ $\Re^{n} \times \Re^{n} \times \Re^{m}$ of the closed-loop system (9), (10), (3), (1) with initial condition $z\left(t_{0}\right)=z_{0} \in \Re^{n}, T_{r+\tau}\left(t_{0}\right) u=u_{0} \in$ $L^{\infty}\left([-r-\tau, 0] ; \Re^{m}\right), T_{r}\left(t_{0}\right) x=x_{0} \in C^{0}\left([-r, 0] ; \Re^{n}\right)$ satisfies the following inequality for all $t \geq t_{0}$ :

$$
\begin{aligned}
|z(t)|+\left\|T_{r}(t) x\right\|_{r}+\left\|T_{r+\tau}(t) u\right\|_{r+\tau} \\
\quad \leq \tilde{\sigma}\left(\left|z_{0}\right|+\left\|x_{0}\right\|_{r}+\left\|u_{0}\right\|_{r+\tau}, t-t_{0}\right) .
\end{aligned}
$$

Some remarks for the dynamic sampled-data feedback given by (9), (10) are in order before we proceed to the proof of Theorem 2.1.

\section{Remark 2.2:}

a) The dynamic sampled-data controller (9), (10) is timevarying if $k$ is time-varying. If $k$ is $T$-periodic then the dynamic sampled-data controller (9), (10) is $T$-periodic too.

b) Since the output $y(t)$ given by (3) of system (1) satisfies the system of differential equations $\dot{y}(t)=f(y(t), u(t-r-\tau))$, for all $t \geq t_{0}+r$, where $t_{0} \geq 0$ is the initial time, we can in principle apply the predictor-based delay compensation approach described in [24] (extended for time-varying feedback laws), which gives the static feedback law $u(t)=k\left(t+\tau, \Phi\left(y(t), T_{r+\tau}(t) u\right)\right)$. This is the inspiration for the construction of the sampled-data dynamic feedback (9), (10): for all $t=t_{0}+i T$, where $i \in Z^{+}$, the value of $u(t)$ computed by (9), (10) is exactly $u(t)=k\left(t+\tau, \Phi\left(y(t), \breve{T}_{r+\tau}(t) u\right)\right)$. However, there are technical problems with the application of the static feedback law $u(t)=k\left(t+\tau, \Phi\left(y(t), \breve{T}_{r+\tau}(t) u\right)\right)$ : given $T_{r}\left(t_{0}\right) x=x_{0} \in C^{0}\left([-r, 0] ; \Re^{n}\right), T_{r+\tau}\left(t_{0}\right) u=u_{0} \in$ $L^{\infty}\left([-r-\tau, 0] ; \Re^{m}\right)$ (initial conditions), we cannot guarantee existence of a measurable and essentially bounded $u:\left[t_{0}-r-\tau, t_{0}+r\right] \rightarrow \Re^{m}$ satisfying the integral equation $u(t)=k\left(t+\tau, \Phi\left(x(t-r), \breve{T}_{r+\tau}(t) u\right)\right)$ for all $t \in\left(t_{0}, t_{0}+r\right]$. For the case $\tau=0$, one sufficient condition for the existence of a solution of the integral equation is that the initial condition $T_{r}\left(t_{0}\right) x=x_{0} \in$ $C^{0}\left([-r, 0] ; \Re^{n}\right), T_{r}\left(t_{0}\right) u=u_{0} \in L^{\infty}\left([-r, 0] ; \Re^{m}\right)$ satisfies the equation $\dot{x}(t-r)=f(x(t-r), u(t-r))$ for all $t \in\left[t_{0}, t_{0}+r\right]:$ in this case the solution is $u(t)=k(t, z(t))$ for $t \in\left(t_{0}, t_{0}+r\right]$, where $z(t)$ is the solution of the initial value problem $\dot{z}(t)=f(z(t), k(t, z(t)))$ with $z\left(t_{0}\right)=x\left(t_{0}\right)$. Other restrictive sufficient conditions for the existence of a solution of the integral equation can be obtained by using fixed point theory. The proof of Theorem 2.1 shows that this issue can be completely avoided for the dynamic sampled-data feedback (9), (10).

c) For every initial condition the value of $u(t)$ computed by (9), (10) is exactly $u(t)=k\left(t+\tau, \Phi\left(y(t), \breve{T}_{r+\tau}(t) u\right)\right)$ for all $t \geq t_{0}+i T$ with $i \in Z^{+}$satisfying $i T \geq r$, so our dynamic sampled-data feedback is based on the predictor principle.

d) For the implementation of the controller (9), (10), we must know the "predictor" mapping $\Phi: \Re^{n} \times$ $L^{\infty}\left([-r-\tau, 0) ; \Re^{m}\right) \rightarrow \Re^{n}$. This mapping can be explicitly computed for

i) Linear systems $\dot{x}=A x+B u$, with $x \in \Re^{n}, u \in \Re^{m}$. In this case (Corollary 3.4 below) the predictor mapping $\Phi: \Re^{n} \times L^{\infty}\left([-r-\tau, 0) ; \Re^{m}\right) \rightarrow \Re^{n}$ is given by the equation $\Phi(x, u):=\exp (A(\tau+r)) x+$ $\int_{-r-\tau}^{0} \exp (-A w) B u(w) d w$.

ii) Bilinear systems $\dot{x}=A x+B u+u C x$, with $x \in \Re^{n}, u \in \Re$ and $A C=C A$. In this case the predictor mapping $\Phi$ : $\Re^{n} \times L^{\infty}\left([-r-\tau, 0) ; \Re^{m}\right) \rightarrow \Re^{n}$ is given by the explicit equation

$$
\begin{aligned}
\Phi(x, u):= & \exp (A(\tau+r)) \exp \left(C \int_{-r-\tau}^{0} u(s) d s\right) x \\
& +\int_{-r-\tau}^{0} \exp (-A w) \exp \left(C \int_{w}^{0} u(s) d s\right) B u(w) d w
\end{aligned}
$$

iii) Nonlinear systems of the following form:

$$
\begin{aligned}
\dot{x}_{1} & =a_{1}(u) x_{1}+f_{1}(u) \\
\dot{x}_{2}= & a_{2}\left(u, x_{1}\right) x_{2}+f_{2}\left(u, x_{1}\right) \\
& \vdots \\
\dot{x}_{n} & =a_{n}\left(u, x_{1}, \ldots, x_{n-1}\right) x_{n}+f_{n}\left(u, x_{1}, \ldots, x_{n-1}\right) \\
x & =\left(x_{1}, \ldots, x_{n}\right)^{\prime} \in \Re^{n}, u \in \Re^{m}
\end{aligned}
$$

where all mappings $a_{i}, f_{i}(i=1, \ldots, n)$ are locally Lipschitz. In this case the predictor mapping $\Phi: \Re^{n} \times L^{\infty}\left([-r-\tau, 0) ; \Re^{m}\right) \rightarrow \Re^{n}$ 
can be constructed inductively. For example, for $n=1$ the predictor mapping is given by

$$
\begin{aligned}
\Phi(x, u)=\exp & \left(\int_{-r-\tau}^{0} a_{1}(u(s)) d s\right) x \\
& +\int_{-r-\tau}^{0} \exp \left(\int_{w}^{0} a_{1}(u(s)) d s\right) f_{1}(u(w)) d w .
\end{aligned}
$$

Example 2.4 below applies Theorem 2.1 to a three-dimensional nonlinear system of the above class. Moreover, the nonholonomic integrator (4) belongs to the above class and Theorem 2.1 can be applied (see Corollary 4.1).

iv) Nonlinear systems $\dot{x}=f(x, u)$, for which there exists a global diffeomorphism $\Theta: \Re^{n} \rightarrow \Re^{n}$ such that the change of coordinates $z=\Theta(x)$ transforms the system to one of the above cases (Corollary 3.7 below).

For globally Lipschitz systems, one can utilize approximate "predictor" mappings $\Phi: \Re^{n} \times L^{\infty}\left([-r-\tau, 0) ; \Re^{m}\right) \rightarrow \Re^{n}$ as shown in [19] under additional and more restrictive hypotheses.

Proof of Theorem 2.1: We start with the following claim, which we prove in the Appendix.

Claim 1: There exists a function $G \in K_{\infty}$ such that for every $t_{0} \geq 0,\left(x_{0}, z_{0}, u_{0}\right) \in C^{0}\left([-r, 0] ; \Re^{n}\right) \times \Re^{n} \times$ $L^{\infty}\left([-r-\tau, 0] ; \Re^{m}\right)$ the solution $(x(t), z(t), u(t)) \quad \in$ $\Re^{n} \times \Re^{n} \times \Re^{m}$ of the closed-loop system (9), (10), (3), (1) with initial condition $z\left(t_{0}\right)=z_{0} \in \Re^{n}, T_{r+\tau}\left(t_{0}\right) u=u_{0} \in$ $L^{\infty}\left([-r-\tau, 0] ; \Re^{m}\right), T_{r}\left(t_{0}\right) x=x_{0} \in C^{0}\left([-r, 0] ; \Re^{n}\right)$ exists for all $t \in\left[t_{0}, t_{0}+T\right]$ and satisfies

$$
\begin{aligned}
& \left\|T_{r+\tau}(t) u\right\|_{r+\tau}+|z(t)|+\left\|T_{r}(t) x\right\|_{r} \\
& \quad \leq G\left(\left|z_{0}\right|+\left\|x_{0}\right\|_{r}+\left\|u_{0}\right\|_{r+\tau}\right) \\
& \quad \text { for all } t \in\left[t_{0}, t_{0}+T\right] .
\end{aligned}
$$

By virtue of induction and Claim 1, the following claim holds.

Claim 2: There exists a function $G \in K_{\infty}$ such that for every $t_{0} \geq 0, p \in Z^{+}, p \geq 1$, and $\left(x_{0}, z_{0}, u_{0}\right) \in C^{0}\left([-r, 0] ; \Re^{n}\right) \times$ $\Re^{n} \times L^{\infty}\left([-r-\tau, 0] ; \Re^{m}\right)$ the solution $(x(t), z(t), u(t)) \in$ $\Re^{n} \times \Re^{n} \times \Re^{m}$ of the closed-loop system (9), (10), (3), (1) with initial condition $z\left(t_{0}\right)=z_{0} \in \Re^{n}, T_{r+\tau}\left(t_{0}\right) u=u_{0} \in$ $L^{\infty}\left([-r-\tau, 0] ; \Re^{m}\right), T_{r}\left(t_{0}\right) x=x_{0} \in C^{0}\left([-r, 0] ; \Re^{n}\right)$ exists for all $t \in\left[t_{0}, t_{0}+p T\right]$ and satisfies

$$
\begin{aligned}
& \left\|T_{r+\tau}(t) u\right\|_{r+\tau}+|z(t)|+\left\|T_{r}(t) x\right\|_{r} \\
& \quad \leq G^{(p)}\left(\left|z_{0}\right|+\left\|x_{0}\right\|_{r}+\left\|u_{0}\right\|_{r+\tau}\right), \text { for all } \\
& \quad t \in\left[t_{0}, t_{0}+p T\right]
\end{aligned}
$$

where $G^{(p)}(s):=\underbrace{G \circ \cdots \circ G}_{p \text { times }}$.

We notice that for all $t \geq t_{0}+i T$ with $i \in Z^{+}$satisfying $i T \geq r$ the solution $(x(t), z(t), u(t)) \in \Re^{n} \times \Re^{n} \times \Re^{m}$ of the closed-loop system (9), (10), (3), (1) satisfies

$$
z(t)=x(t+\tau)
$$

Consequently, for all $t \geq t_{0}+i T+\tau$ with $i \in Z^{+}$satisfying $i T \geq r$ it holds that

$$
u(t-\tau)=k(t, x(t))
$$

Hypothesis (H2) in conjunction with inequality (8) and (15) implies that the following inequality holds:

$$
\begin{array}{r}
|x(t)| \leq \sigma\left(\left|x\left(t_{0}+i T+\tau\right)\right|, t-t_{0}-i T-\tau\right) \forall t \\
\geq t_{0}+i T+\tau .
\end{array}
$$

Define $p=[r / T]+[\tau / T]+2$. Using (13), (14) and (16), it follows that the following inequality holds for all $t \geq t_{0}+p T$ :

$$
\begin{aligned}
& |x(t)|+|z(t)| \\
& \quad \leq 2 \sigma\left(G ^ { ( p ) } \left(\left\|T_{r+\tau}\left(t_{0}\right) u\right\|_{r+\tau}\right.\right. \\
& \left.\left.\quad+\left|z\left(t_{0}\right)\right|+\left\|T_{r}\left(t_{0}\right) x\right\|_{r}\right), t-t_{0}-p T\right) .
\end{aligned}
$$

Define $\quad \tilde{\sigma}(s, t) \quad:=\quad 2 \sigma\left(G^{(p)}(s), t-p T-r\right)$ $+g\left(2 \sigma\left(G^{(p)}(s), t-p T-r\right)\right)$ for all $t \geq p T+r$ and $\tilde{\sigma}(s, t):=2 \sigma\left(G^{(p)}(s), 0\right)+g\left(2 \sigma\left(G^{(p)}(s), 0\right)\right)$ for all $t \in[0, p T+r)$. Using (7), (14), (15) and (17) we can conclude that (11) holds. The proof is complete $\triangleleft$.

Remark 2.3: The proof of Theorem 2.1 shows that if $k \in$ $C^{1}\left(\Re^{+} \times \Re^{n} ; \Re^{m}\right)$ with $k(t, 0)=0$ for all $t \geq 0$, is a nonuniform in time stabilizer for system (see [13]) then we can prove that the closed-loop system (9), (10), (3), (1) is non-uniformly in time Globally Asymptotically Stable, i.e., there exist functions $\tilde{\sigma} \in K L$ and a positive continuous function $\beta: \Re^{+} \rightarrow(0,+\infty)$ such that for every $t_{0} \geq 0,\left(x_{0}, z_{0}, u_{0}\right) \in C^{0}\left([-r, 0] ; \Re^{n}\right) \times$ $\Re^{n} \times L^{\infty}\left([-r-\tau, 0] ; \Re^{m}\right)$, the solution $(x(t), z(t), u(t)) \in$ $\Re^{n} \times \Re^{n} \times \Re^{m}$ of the closed-loop system (9), (10), (3), and (1) with initial condition $z\left(t_{0}\right)=z_{0} \in \Re^{n}, T_{r+\tau}\left(t_{0}\right) u=u_{0} \in$ $L^{\infty}\left([-r-\tau, 0] ; \Re^{m}\right), T_{r}\left(t_{0}\right) x=x_{0} \in C^{0}\left([-r, 0] ; \Re^{n}\right)$ satisfies the following inequality for all $t \geq t_{0}$ :

$$
\begin{aligned}
|z(t)|+ & \left\|T_{r}(t) x\right\|_{r}+\left\|T_{r+\tau}(t) u\right\|_{r+\tau} \\
& \leq \tilde{\sigma}\left(\beta\left(t_{0}\right)\left(\left|z_{0}\right|+\left\|x_{0}\right\|_{r}+\left\|u_{0}\right\|_{r+\tau}\right), t-t_{0}\right)
\end{aligned}
$$

In this case, there is no need to assume that inequality (7) holds.

We next present an example which shows how the obtained results can be applied to feedforward nonlinear systems.

Example 2.4 (Control of Strict-Feedforward Systems With Arbitrarily Sparse Sampling): Consider the following example taken from [24]:

$$
\begin{aligned}
\dot{x}_{1}(t) & =x_{2}(t)+x_{3}^{2}(t) \\
\dot{x}_{2}(t) & =x_{3}(t)+x_{3}(t) u(t-\tau) \\
\dot{x}_{3}(t) & =u(t-\tau) \\
x(t) & =\left(x_{1}(t), x_{2}(t), x_{3}(t)\right)^{\prime} \in \Re^{3}, u(t) \in \Re .
\end{aligned}
$$

Here, we consider the stabilization problem for (19) with output given by (3) available only at the discrete time instants $\tau_{i}$ (the sampling times) with $\tau_{i+1}-\tau_{i}=T>0$, where $T>0$ is the 
sampling period. Hypothesis (H1) holds for system (19) and the predictor mapping can be explicitly expressed by the equations

$$
\begin{aligned}
& \Phi(x, u):= \\
& {\left[\begin{array}{c}
\left.x_{2}+\delta x_{3}+x_{3} \int_{-\delta}^{0} u(s) d s+\int_{-\delta}^{0}(1+u(s)) \int_{-\delta}^{s} u(q) d q d s\right] \\
x_{3}+\int_{-\delta}^{0} u(s) d s
\end{array}\right]}
\end{aligned}
$$

where $\delta=r+\tau$ and

$$
\begin{aligned}
\phi_{1}(x, u) & =x_{1}+\delta x_{2}+\delta x_{3}^{2}+\frac{1}{2} \delta^{2} x_{3} \\
+ & 3 x_{3} \int_{-\delta}^{0} \int_{-\delta}^{s} u(q) d q d s \\
+ & \int_{-\delta}^{0} \int_{-\delta}^{s}(1+u(w)) \int_{-\delta}^{w} u(q) d q d w d s \\
+ & \int_{-\delta}^{0}\left(\int_{-\delta}^{s} u(q) d q\right)^{2} d s .
\end{aligned}
$$

Moreover, hypothesis (H2) holds as well with the smooth, timeindependent feedback law

$$
\begin{aligned}
& k(x):=-x_{1}-3 x_{2}-\frac{3}{8} x_{2}^{2}+\frac{3}{4} x_{3}\left(\frac{1}{2} c(x)-4-x_{1}-2 x_{2}\right) \\
& c(x):=x_{3}+x_{2} x_{3}+\frac{5}{4} x_{3}^{2}-\frac{1}{2} x_{3}^{3}-\frac{3}{4}\left(x_{2}-\frac{1}{2} x_{3}^{2}\right)^{2} \cdot
\end{aligned}
$$

It follows from Theorem 2.1 that the dynamic sampled-data controller $u(t)=k(z(t))$ with

$$
\begin{aligned}
\dot{z}_{1}(t) & =z_{2}(t)+z_{3}^{2}(t) \\
\dot{z}_{2}(t) & =z_{3}(t)+z_{3}(t) u(t) \\
\dot{z}_{3}(t) & =u(t), \quad \text { for } t \in\left[\tau_{i}, \tau_{i+1}\right) \\
z(t) & =\left(z_{1}(t), z_{2}(t), z_{3}(t)\right)^{\prime} \in \Re^{3}
\end{aligned}
$$

and

$$
z\left(\tau_{i+1}\right)=\Phi\left(y\left(\tau_{i+1}\right), \breve{T}_{r+\tau}\left(\tau_{i+1}\right) u\right), i \in Z^{+}
$$

where $\Phi: \Re^{3} \times L^{\infty}\left([-r-\tau, 0) ; \Re^{m}\right) \rightarrow \Re^{3}$ is defined by (20), (21) and $k: \Re^{3} \rightarrow \Re$ is defined by (22), guarantees global asymptotic stability for system (19). The reader should notice that the dynamic sampled-data controller (23), (24) can still be used even if no delays are present but the state is available only at the discrete time instants $\tau_{i}$ (the sampling times) with $\tau_{i+1}-\tau_{i}=T>0$, where $T>0$ is the sampling period. Hence, in this section we have provided, as a special case, the first solution to the problem of global asymptotic stabilization of strict feedforward systems with arbitrarily sparse in time sampling of the state and with continuous control $\triangleleft$.

\section{SAMPLED-DATA FEEDBACK FOR INPUT APPLIED WITH ZERO-ORDER HOLD}

\section{A. General Design}

This section is devoted to the case where the input is applied with zero-order hold. In this section, we assume that (2) is globally stabilizable with feedback applied with zero order hold. This is very different from hypothesis (H2) in the previous section.

Hypothesis (H3) (Input Applied With Zero-Order Hold): There exists $k: \Re^{n} \rightarrow \Re^{m}, g \in K_{\infty}, T>0$ such that

$$
|k(x)| \leq g(|x|), \text { for all } x \in \Re^{n}
$$

and such that $0 \in \Re^{n}$ is uniformly globally asymptotically stable for the sampled-data system

$$
\begin{aligned}
\dot{x}(t) & =f\left(x(t), k\left(x\left(\tau_{i}\right)\right)\right), t \in\left[\tau_{i}, \tau_{i+1}\right) \\
x\left(\tau_{i+1}\right) & =\lim _{t \rightarrow \tau_{i+1}^{-}} x(t) \\
\tau_{i+1} & =\tau_{i}+T, \tau_{0}=0 \geq 0, x(0)=x_{0} \in \Re^{n}
\end{aligned}
$$

in the sense that there exists a function $\sigma \in K L$ such that for every $x_{0} \in \Re^{n}$ the solution $x(t)$ of (26) with initial condition $x(0)=x_{0} \in \Re^{n}$ satisfies inequality (8) with $t_{0}=0$ for all $t \geq 0$.

Remark 3.1: Hypothesis (H3) seems like a restrictive hypothesis, because it demands global stabilizability by means of sampled-data feedback with positive sampling rate. However, hypothesis (H3) can be satisfied for the following:

i) Linear stabilizable systems, $\dot{x}=A x+B u$, where $A \in$ $\Re^{n \times n}, B \in \Re^{n \times m}$ (see Corollary 3.4 and Remark 3.5 below).

ii) Nonlinear systems of the form $\dot{x}=f(x)+g(x) u, x \in$ $\Re^{n}, u \in \Re$, where the vector field $f: \Re^{n} \rightarrow \Re^{n}$ is globally Lipschitz and the vector field $g: \Re^{n} \rightarrow \Re^{n}$ is locally Lipschitz and bounded, which can be stabilized by a globally Lipschitz feedback law $u=k(x)$ (see [11]).

iii) Nonlinear systems of the form $\dot{x}_{i}=f_{i}(x, u)+$ $g_{i}(x, u) x_{i+1}$ for $i=1, \ldots, n-1$ and $\dot{x}_{n}=$ $f_{n}(x, u)+g_{n}(x, u) u$, where the drift terms $f_{i}(x, u)$ $(i=1, \ldots, n)$ satisfy the linear growth conditions $\left|f_{i}(x, u)\right| \leq L\left|x_{1}\right|+\cdots+L\left|x_{i}\right|(i=1, \ldots, n)$ for certain constant $L \geq 0$ and there exist constants $b \geq a>0$ such that $a \leq g_{i}(x, u) \leq b$ for all $i=1, \ldots, n, x \in \Re^{n}$, $u \in \Re$ (see [16]).

iv) Asymptotically controllable homogeneous systems with positive minimal power and zero degree (see [8]).

v) Systems satisfying the reachability hypotheses of Theorem 3.1 in [18], or hypotheses (69), (70), (71) in Section IV of [15].

vi) Nonlinear systems $\dot{x}=f(x, u)$, for which there exists a global diffeomorphism $\Theta: \Re^{n} \rightarrow \Re^{n}$ such that the change of coordinates $z=\Theta(x)$ transforms the system to one of the above cases. 
Consider system (1) under hypotheses (H1), (H3) for system (2). In this case we propose a feedback law that is simply a composition of the feedback stabilizer and the delay compensator:

$$
u(t)=k\left(\Phi\left(y\left(\tau_{i}\right), \breve{T}_{r+\tau}\left(\tau_{i}\right) u\right)\right), t \in\left[\tau_{i}, \tau_{i+1}\right)
$$

where $\tau_{i}=i T, i \in Z^{+}$are the sampling times and $\Phi: \Re^{n} \times$ $L^{\infty}\left([-r-\tau, 0) ; \Re^{m}\right) \rightarrow \Re^{n}$ is the predictor mapping involved in (5), (6). The control action is applied with zero order hold, i.e., it is constant on $\left[\tau_{i}, \tau_{i+1}\right)$; however, the control action affecting system (1) remains constant on the interval $\left[\tau_{i}+\tau, \tau_{i+1}+\tau\right)$. Our main result is stated next.

Theorem 3.2: Let $T>0, r, \tau \geq 0$ with $r+\tau>0$ be given. Moreover, suppose that hypotheses (H1), (H3) hold for system (2). Then the closed-loop system (1) with (27), i.e., the following sampled-data system:

$$
\begin{aligned}
\dot{x}(t) & =f(x(t), u(t-\tau)) \\
u(t) & =k\left(\Phi\left(x\left(\tau_{i}-r\right), \breve{T}_{r+\tau}\left(\tau_{i}\right) u\right)\right), t \in\left[\tau_{i}, \tau_{i+1}\right), i \in Z^{+} \\
\tau_{i+1} & =\tau_{i}+T, \tau_{0}=0
\end{aligned}
$$

is uniformly globally asymptotically stable, in the sense that there exists a function $\tilde{\sigma} \in K L$ such that for every $\left(x_{0}, u_{0}\right) \in C^{0}\left([-r, 0] ; \Re^{n}\right) \times L^{\infty}\left([-r-\tau, 0) ; \Re^{m}\right)$, the solution $(x(t), u(t)) \in \Re^{n} \times \Re^{m}$ of system (28) with initial condition $\widetilde{T}_{r+\tau}(0) u=u_{0} \in L^{\infty}\left([-r-\tau, 0) ; \Re^{m}\right)$, $T_{r}(0) x=x_{0} \in C^{0}\left([-r, 0] ; \Re^{n}\right)$ satisfies the following inequality for all $t \geq 0$ :

$$
\left\|T_{r}(t) x\right\|_{r}+\left\|\breve{T}_{r+\tau}(t) u\right\|_{r+\tau} \leq \tilde{\sigma}\left(\left\|x_{0}\right\|_{r}+\left\|u_{0}\right\|_{r+\tau}, t\right) .
$$

Finally, if system (26) satisfies the dead-beat property of order $j T$, where $j \in Z^{+}$is positive, i.e., for all $x_{0} \in \Re^{n}$ the solution $x(t)$ of (26) with initial condition $x(0)=x_{0} \in \Re^{n}$ satisfies $x(t)=0$ for all $t \geq j T$ then system (28) satisfies the dead-beat property of order $(j+p) T$, where $p=[r / T]+[\tau / T]+2$, i.e., for every $\left(x_{0}, u_{0}\right) \in C^{0}\left([-r, 0] ; \Re^{n}\right) \times L^{\infty}\left([-r-\tau, 0) ; \Re^{m}\right)$, the solution $(x(t), u(t)) \in \Re^{n} \times \Re^{m}$ of system (28) with initial condition $\breve{T}_{r+\tau}(0) u=u_{0} \in L^{\infty}\left([-r-\tau, 0) ; \Re^{m}\right), T_{r}(0) x=$ $x_{0} \in C^{0}\left([-r, 0] ; \Re^{n}\right)$ satisfies $x(t)=0$ for all $t \geq(j+p) T$.

Remark 3.3: If we denote $T_{1} \geq 0$ to be the delay in receiving the measured data, $T_{2} \geq 0$ the computation time for the quantity $v=k(\Phi(x, u))$, where $(x, u) \in \Re^{n} \times L^{\infty}\left([-r-\tau, 0) ; \Re^{m}\right)$, and $T_{3} \geq 0$ the time for the data to reach the actuator, then one should notice that $r=T_{1}+T_{2}$ and $\tau=T_{3}$.

Proof of Theorem 3.2: Using the Fact, we can guarantee the existence of $b \in K_{\infty}$ such that for every $y \in \Re^{n}, u \in$ $L^{\infty}\left([-\tau, T-\tau) ; \Re^{m}\right)$ the solution $x(t)$ of (1) with initial condition $x(0)=y \in \Re^{n}$ corresponding to input $u \in L^{\infty}([-\tau, T-$ $\left.\tau) ; \Re^{m}\right)$ satisfies

$$
|x(t)| \leq b\left(|y|+\sup _{-\tau \leq s<t-\tau}|u(s)|\right), \text { for all } t \in(0, T] .
$$

We next continue with the following claim. Its proof is provided in the Appendix.
Claim 3: For every $p \in Z^{+}, p \geq 1$, there exists a function $G_{p} \in K_{\infty}$ such that for every $\left(x_{0}, u_{0}\right) \in$ $C^{0}\left([-r, 0] ; \Re^{n}\right) \times L^{\infty}\left([-r-\tau, 0) ; \Re^{m}\right), \quad$ the solution $(x(t), u(t)) \in \Re^{n} \times \Re^{m}$ of system (28) with initial condition $\breve{T}_{r+\tau}(0) u=u_{0} \in L^{\infty}\left([-r-\tau, 0) ; \Re^{m}\right)$, $T_{r}(0) x=x_{0} \in C^{0}\left([-r, 0] ; \Re^{n}\right)$ exists for all $t \in[0, p T]$ and satisfies

$$
\begin{aligned}
& \left\|\breve{T}_{r+\tau}(t) u\right\|_{r+\tau}+\left\|T_{r}(t) x\right\|_{r} \\
& \quad \leq G_{p}\left(\left\|u_{0}\right\|_{r+\tau}+\left\|x_{0}\right\|_{r}\right) \text { for all } t \in[0, p T] .
\end{aligned}
$$

We next notice that for all $i \in Z^{+}$with $i T \geq r$ the solution $(x(t), u(t)) \in \Re^{n} \times \Re^{m}$ of system (28) satisfies

$$
u(t-\tau)=k(x(i T+\tau)), \forall t \in[i T+\tau,(i+1) T+\tau) .
$$

Hypothesis (H3) in conjunction with inequality (8) with $t_{0}=$ 0 and (32) implies that the following inequality holds for all $i \in Z^{+}$with $i T \geq r$

$$
|x(t)| \leq \sigma(|x(i T+\tau)|, t-i T-\tau), \forall t \geq i T+\tau
$$

Define $p=[r / T]+[\tau / T]+2$. Using (31) and (33), we conclude that the following inequality holds:

$$
|x(t)| \leq \sigma\left(G_{p}\left(\left\|u_{0}\right\|_{r+\tau}+\left\|x_{0}\right\|_{r}\right), t-p T\right) \forall t \geq p T .
$$

Define

$$
\begin{aligned}
\tilde{\sigma}(s, t):=\sigma\left(G_{p}(s), t-(p+1) T-r\right) \\
\\
+
\end{aligned}
$$

for $t \geq(p+1) T+r$ and

$\tilde{\sigma}(s, t):=\exp ((p+1) T+r-t) \sigma\left(G_{p}(s), 0\right)+g\left(\sigma\left(G_{p}(s), 0\right)\right)$

for all $t \in[0,(p+1) T+r)$. Using (25), (31), (32), (33) and (34) we conclude that (29) holds. Notice that if $\sigma(s, t)=0$ for all $s \geq 0$ and $t \geq j T$ (i.e., the dead-beat property of order $j T$ ) then (34) implies that $x(t)=0$ for all $t \geq(j+p) T$ (i.e., the dead-beat property of order $(j+p) T)$. The proof is complete.

Theorem 3.2 can be applied to all forward complete systems satisfying Remark 2.2(d) and Remark 3.1. Here we focus on two special cases: the case of stabilizable linear time invariant (LTI) systems and the case of systems which are diffeomorphically equivalent to a chain of integrators (DECI). The latter case includes the linearizable strict feedforward systems (see [22]).

\section{B. Design for Stabilizable LTI Systems}

For the LTI case, there are matrices $A \in \Re^{n \times n}, B \in \Re^{n \times m}$ such that $f(x, u)=A x+B u$. In this case the predictor mapping is given by the explicit expression $\Phi(x, u)=\exp (A(r+\tau)) x+$ $\int_{-r-\tau}^{0} \exp (-A s) B u(s) d s$. The linear feedback law $k(x)=K x$, where $K \in \Re^{m \times n}$, satisfies hypothesis (H3) if and only if there exists $T>0$ such that all the eigenvalues of the matrix $\exp (A T)\left(I+\int_{0}^{T} \exp (-A w) d w B K\right)$ are strictly inside the unit circle on the complex plane. 
The use of the sampled-data feedback law $u_{i}=K y_{i}$, where $u_{i}=u(i T)$ and $y_{i}=y(i T)$, does not in general guarantee global asymptotic stability for the delayed case, where $r+\tau>$ 0 . However, Theorem 3.2 can be used for the design of a delay compensator, which guarantees global asymptotic stability for the corresponding closed-loop system. The following corollary is a direct consequence of Theorem 3.2 and its proof is omitted.

Corollary 3.4 (Stabilization of Linear Networked Control Systems With Delays): Let $T>0, r, \tau \geq 0$ with $r+\tau>0$ be given and let $l \in Z^{+}$such that $\tau+r=l T+\delta$ with $0 \leq \delta<T$. Moreover, suppose that all the eigenvalues of the matrix $\exp (A T)\left(I+\int_{0}^{T} \exp (-A w) d w B K\right)$ are strictly inside the unit circle on the complex plane. Then the closed-loop LTI system

$$
\begin{aligned}
& \dot{x}(t)=A x(t)+B u(t-\tau) \\
& u(t)=u_{i}, t \in[i T,(i+1) T), i \in Z^{+}
\end{aligned}
$$

with input applied with zero order hold given by

$$
u_{i}=K \exp (A(r+\tau)) y_{i}+K \sum_{j=1}^{l+1} Q_{j} B u_{i-j}
$$

where $y_{i}=y(i T)=x(i T-r)$ and

$$
\begin{aligned}
Q_{j} & =\exp (A j T) \int_{0}^{T} \exp (-A s) d s, j=1, \ldots, l \\
Q_{l+1} & =\exp (A l T)\left(\int_{0}^{\delta} \exp (A s) d s\right)
\end{aligned}
$$

is globally exponentially stable, in the sense that there exist constants $M, \sigma>0$ such that for every $\left(x_{0}, u_{0}\right) \in$ $C^{0}\left([-r, 0] ; \Re^{n}\right) \times L^{\infty}\left([-r-\tau, 0) ; \Re^{m}\right)$, the solution $(x(t), u(t)) \in \Re^{n} \times \Re^{m}$ of system (35), (36) with initial condition $\breve{T}_{r+\tau}(0) u=u_{0} \in L^{\infty}\left([-r-\tau, 0) ; \Re^{m}\right)$, $T_{r}(0) x=x_{0} \in C^{0}\left([-r, 0] ; \Re^{n}\right)$ satisfies the following inequality for all $t \geq 0$ :

$$
\begin{aligned}
\left\|T_{r}(t) x\right\|_{r}+\left\|\widetilde{T}_{r+\tau}(t) u\right\|_{r+\tau} \\
\quad \leq M\left(\left\|x_{0}\right\|_{r}+\left\|u_{0}\right\|_{r+\tau}\right) \exp (-\sigma t) .
\end{aligned}
$$

Remark 3.5: If the pair of matrices $(A, B)$ is stabilizable then there exists $K \in \Re^{m \times n}$ such that the matrix $(A+B K)$ is Hurwitz, a symmetric positive definite matrix $P \in \Re^{n \times n}$ and a constant $\mu>0$ such that $P(A+B K)+(A+B K)^{\prime} P+\mu I<0$. Using Corollary 4.3 in [16] with $V(x)=x^{\prime} P x, a(s):=\lambda s$, arbitrary $\lambda \in(0,1)$, one can show that all the eigenvalues of the matrix $\exp (A T)\left(I+\int_{0}^{T} \exp (-A w) d w B K\right)$ are strictly inside the unit circle on the complex plane for all $T>0$ satisfying

$$
2 \frac{T\left(|A| \sqrt{\frac{k_{2}}{k_{1}}}+|B K|\right)}{1-T|B K|}|P B K|<\mu .
$$

where $k_{2}=\max \left\{x^{\prime} P x:|x|=1\right\}$ and $k_{2}=\min \left\{x^{\prime} P x:|x|=1\right\}$. Of course, the estimate for the maximum allowable sampling period provided by the above inequalities is conservative in most cases. Other estimates for the maximum allowable sampling period can be found in [37].

1) Example 3.6: We consider the scalar control system

$$
\dot{x}(t)=x(t)+u(t)
$$

where $x(t) \in \Re, u(t) \in \Re$. The system can be exponentially stabilized by the linear feedback $u=-k x$ with $k>1$ applied with zero order hold, i.e.,

$$
\begin{aligned}
u(t) & =u_{i}, t \in[i T,(i+1) T) \\
u_{i} & =-k x(i T) \quad i \in Z^{+}
\end{aligned}
$$

where the sampling period $T>0$ must satisfy

$$
T<\ln \left(1+\frac{2}{k-1}\right) \text {. }
$$

The use of the same feedback law for the case where measurement delays are present is described by the equations

$$
\begin{aligned}
\dot{x}(t) & =x(t)+u(t) \\
u(t) & =u_{i}, t \in[i T,(i+1) T) \\
u_{i} & =-k x(i T-r), i \in Z^{+}
\end{aligned}
$$

where $r \geq 0$ is the measurement delay. Numerical experiments for the closed-loop system (42) show that for each pair of $k>1$ and $T>0$ satisfying (41), there exists $r_{c}>0$ such that:

- if $r<r_{c}$ then system (42) is globally exponentially stable;

- if $r>r_{c}$ then system (42) admits exponentially growing solutions.

For the case $k=2, T=1$ the value of the critical measurement delay satisfies $r_{c} \in(0.20,0.21)$. Fig. 1 shows the evolution of the state for system (42) with $k=2, T=1, r=0.1$ and initial condition $x(\theta)=1$ for $\theta \in[-1,0]$. The state converges exponentially to zero. Fig. 2 shows the evolution of the state for system (42) with $k=2, T=1, r=0.3$ and same initial condition $x(\theta)=1$ for $\theta \in[-1,0]$. In this case, the state grows exponentially, indicating instability.

It is clear that for the case $r>r_{c}$ one needs a delay compensator. Notice that for the case $k=2, T=1$ the critical measurement delay $r_{c} \in(0.20,0.21)$ is only a small fraction of the sampling period. The usual practice would be to ignore the delay and this would give rise to completely unacceptable results. Corollary 3.4 shows that the feedback law

$$
\begin{aligned}
u(t) & =u_{i}, t \in[i T,(i+1) T) \\
u_{i} & =-k \exp (r) x(i T-r)-k(\exp (r)-1) u_{i-1}
\end{aligned}
$$

will guarantee global exponential stability for the closed-loop system (39) with (43) when $r<T$. Indeed, Fig. 3 shows the evolution of the state for the closed-loop system (39) with (43), $k=2, T=1, r=0.3$ and initial condition $x(\theta)=1$ for $\theta \in[-1,0], u(\theta)=4$ for $\theta \in[-1,0)$. The state converges exponentially to zero. 


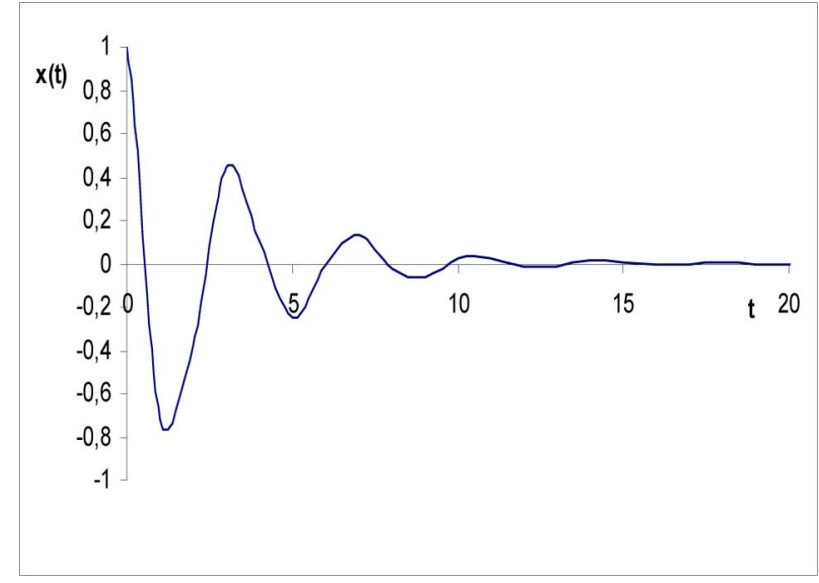

Fig. 1. Evolution of the state for system (42) with $k=2, T=1, r=0.1$ and initial condition $x(\theta)=1$ for $\theta \in[-1,0]$.

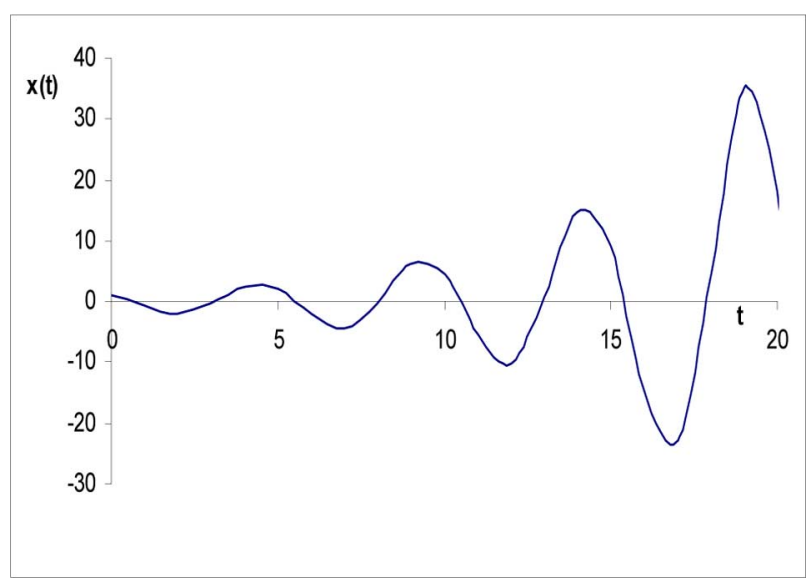

Fig. 2. Evolution of the state for system (42) with $k=2, T=1, r=0.3$ and initial condition $x(\theta)=1$ for $\theta \in[-1,0]$.

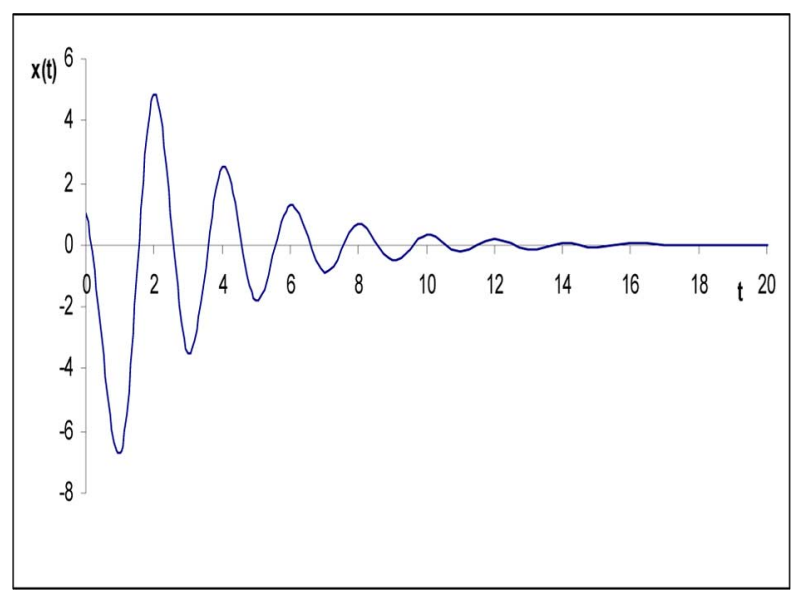

Fig. 3. Evolution of the state for the closed-loop system (39) with (43), $k=2$, $T=1, r=0.3$ and initial condition $x(\theta)=1$ for $\theta \in[-1,0], u(\theta)=4$ for $\theta \in[-1,0)$.

This example demonstrates that the delay-compensating predictor-based feedback (43) extends the range of measurement delays for which stabilization is achieved for given $k>1$ and $T>0$ satisfying (41). $\triangleleft$

\section{Design for Controllable Systems Linearizable by Coordinate Change}

The class of systems that are Diffeomorphically Equivalent to a Chain of Integrators (DECI) is the class of all nonlinear systems $\dot{x}=f(x, u), x \in \Re^{n}, u \in \Re$, where $f: \Re^{n} \times \Re \rightarrow \Re^{n}$ is locally Lipschitz with $f(0,0)=0$, for which there exists a global diffeomorphism $\Theta: \Re^{n} \rightarrow \Re^{n}$ such that the change of coordinates $z=\Theta(x)$ transforms the system to the linear system $\dot{z}=A_{0} z+B_{0} u$, where $B_{0}^{\prime}=(0, \ldots, 0,1), A_{0}=$ $\left\{a_{i, j}, i, j=1, \ldots, n\right\}$ with $a_{i, i+1}=1$ for $i=1, \ldots, n-1$ and $a_{i, j}=0$ if $j \neq i+1$.

In this case, for every $T>0$ there exists $K \in \Re^{n}$, such that all the eigenvalues of the matrix $\exp \left(A_{0} T\right)\left(I+\int_{0}^{T} \exp \left(-A_{0} w\right) d w B_{0} K^{\prime}\right)$ are zero. For example, for $n=2$ the vector $K \in \Re^{2}$ is defined by $K^{\prime}=-\left(1 / T^{2}, 3 / 2 T\right)$. If all eigenvalues of the matrix $\exp \left(A_{0} T\right)\left(I+\int_{0}^{T} \exp \left(-A_{0} w\right) d w B_{0} K^{\prime}\right)$ are zero then the sampled-data controller with zero order hold

$$
u(t)=K^{\prime} z(i T), t \in[i T,(i+1) T), i \in Z^{+}
$$

applied to the linear system $\dot{z}=A_{0} z+B_{0} u$ will guarantee the dead-beat property of order $n T$ for the resulting closed-loop system, i.e., $z(t)=0$, for all $t \geq n T$ and for all initial conditions $z(0) \in \Re^{n}$. Thus, we can conclude that the sampled-data controller with zero order hold

$$
u(t)=K^{\prime} \Theta(x(i T)), t \in[i T,(i+1) T), i \in Z^{+}
$$

applied to the nonlinear system $\dot{x}=f(x, u)$ will guarantee the dead-beat property of order $n T$ for the resulting closed-loop system. Therefore, Theorem 3.2 and Corollary 3.4 lead us to the following corollary.

Corollary 3.7 (Predictor for Linearizable Controllable Systems): Let $T>0, r, \tau \geq 0$ with $r+\tau>0$ be given and let $l \in Z^{+}$such that $\tau+r=l T+\delta$ with $0 \leq \delta<T$. Consider system (1) with $m=1$ and suppose that there exists a global diffeomorphism $\Theta: \Re^{n} \rightarrow \Re^{n}$ such that

$$
D \Theta(x) f(x, u)=A_{0} \Theta(x)+B_{0} u, \text { for all } x \in \Re^{n}, u \in \Re
$$

where $D \Theta(x)$ is the Jacobian of $\Theta, B_{0}^{\prime}=(0, \ldots, 0,1)$, $A_{0}=\left\{a_{i, j}, i, j=1, \ldots, n\right\}$ with $a_{i, i+1}=1$ for $i=1, \ldots, n-1$ and $a_{i, j}=0$ if $j \neq i+1$. Let $K \in \Re^{n}$ be such that all eigenvalues of the matrix $\exp \left(A_{0} T\right)\left(I+\int_{0}^{T} \exp \left(-A_{0} w\right) d w B_{0} K^{\prime}\right)$ are strictly inside the unit circle on the complex plane. Then the closed-loop system (2) with input applied with zero order hold given by

$$
\begin{aligned}
u(t)= & u_{i}, t \in[i T,(i+1) T), i \in Z^{+} \\
u_{i}= & K^{\prime} \exp \left(A_{0}(r+\tau)\right) \Theta\left(y_{i}\right) \\
& +K^{\prime} \sum_{j=1}^{l+1} Q_{j} B_{0} u_{i-j}
\end{aligned}
$$


where $y_{i}=y(i T)=x(i T-r)$ and the matrices $Q_{j}(j=1, \ldots, l+1)$ are defined by (37) with $A_{0}$ in place of $A$, is Globally Asymptotically Stable. Moreover, if all eigenvalues of the matrix $\exp \left(A_{0} T\right)\left(I+\int_{0}^{T} \exp \left(-A_{0} w\right) d w B_{0} K^{\prime}\right)$ are zero then for every $\left(x_{0}, u_{0}\right) \in C^{0}\left([-r, 0] ; \Re^{n}\right) \times$ $L^{\infty}\left([-r-\tau, 0) ; \Re^{m}\right)$, the solution $(x(t), u(t)) \in \Re^{n} \times \Re^{m}$ of system (1), (45), (46) with initial condition $\breve{T}_{r+\tau}(0) u=$ $u_{0} \in L^{\infty}\left([-r-\tau, 0) ; \Re^{m}\right), T_{r}(0) x=x_{0} \in C^{0}\left([-r, 0] ; \Re^{n}\right)$ satisfies

$$
x(t)=0, \text { for all } t \geq(l+2+n) T .
$$

Example 3.8: Dead-beat control with a predictor can be applied to any delayed 2-D strict feedforward system, i.e., any system of the form

$$
\dot{x}_{1}(t)=x_{2}(t)+p\left(x_{2}(t)\right) u(t-\tau), \quad \dot{x}_{2}(t)=u(t-\tau)
$$

where $p: \Re \rightarrow \Re$ is a smooth function and the measurements are sampled and given by (3). The diffeomorphism given by (see [22])

$$
\Theta(x)=\left[x_{1}-\int_{0}^{x_{2}} p(w) d w, \quad x_{2}\right]^{\prime}
$$

transforms system (48) with $\tau=0$ to a chain of two integrators. Therefore, the feedback law

$$
u=-\frac{1}{T^{2}} x_{1}+\frac{1}{T^{2}} \int_{0}^{x_{2}} p(w) d w-\frac{3}{2 T} x_{2}
$$

applied with zero order hold and sampling period $T>0$ achieves global stabilization of system (48) with $\tau=0$ when no measurement delays are present. Moreover, the dead-beat property of order $2 T$ is guaranteed for the corresponding closed-loop system. Interestingly, the feedback law (50) is also globally asymptotically stabilizing as a continuous-time controller, placing the closed-loop poles in the $\Theta$-coordinates at $-3 \pm i \sqrt{7} / 4 T$ for any $T>0$. We next consider the case where we have measurement delay $r>0$ satisfying $r<T$. In this case $(l=q=0, \tilde{r}=r)$ we apply Corollary 3.7 and we can conclude that the feedback law (45) with

$$
\begin{aligned}
u_{i}=-\frac{1}{T^{2}} x_{1}\left(\tau_{i}-r\right)+\frac{1}{T^{2}} \int_{0}^{x_{2}\left(\tau_{i}-r\right)} p(w) d w \\
-\frac{3 T+2 r}{2 T^{2}} x_{2}\left(\tau_{i}-r\right)-\frac{r(r+3 T)}{2 T^{2}} u_{i-1}
\end{aligned}
$$

guarantees the dead-beat property of order $3 T$ for the corresponding closed-loop system. Similar formulas to (51) are obtained for other cases, where $\tau>0$ or $r \geq T$. $\triangleleft$

\section{Stabilization of a Nonholonomic Mobile Robot OVER A LONG-DisTANCE COMMUNICATION NETWORK With ARBITRARILY SPARSE SAMPLING}

The reduced-order model of a three-wheeled vehicle with two independent rear motorized wheels can be described by the following system of differential equations:

$$
\dot{x}(t)=v(t) \cos (\theta(t)), \dot{y}(t)=v(t) \sin (\theta(t)), \dot{\theta}(t)=\omega(t)
$$

where $(x, y)$ are the coordinates of the center of mass of the vehicle and $\theta$ is the angle between the axis of the vehicle and the horizontal axis. The inputs $v$ and $\omega$ are linear combinations of the angular velocities of the two rear wheels.

The coordinate transformation

$$
\begin{aligned}
& x_{1}=x \cos (\theta)+y \sin (\theta) \\
& x_{2}=x \sin (\theta)-y \cos (\theta) \\
& x_{3}=\theta
\end{aligned}
$$

and the input transformation

$$
u_{1}=v-x_{2} \omega, \quad u_{2}=\omega
$$

brings system (52) to the form (4). Many researchers have obtained results for the stabilization of the equilibrium point $0 \in$ $\Re^{3}$ of system (4). Here, we assume that the measurements $x(t-$ $r), y(t-r), \theta(t-r)$, where $r>0(i=1,2,3)$, are available at discrete time instants which differ by a constant $T>0$. Moreover, we assume that there is a time delay $\tau \geq 0$ between the computed control action and the applied input (communication delay). In this case the equations of the vehicle are

$$
\begin{aligned}
& \dot{x}(t)=v(t-\tau) \cos (\theta(t)) \\
& \dot{y}(t)=v(t-\tau) \sin (\theta(t)) \\
& \dot{\theta}(t)=\omega(t-\tau)
\end{aligned}
$$

with measurements $x(t-r), y(t-r)$ and $\theta(t-r)$.

The reader should notice that hypotheses (H1), (H2) hold for system (52). Particularly, there exist smooth time-periodic feedback stabilizers for system (4) (see [31], [38]) and consequently we can guarantee that hypothesis (H2) holds. The predictor mapping for system (57) is given by the following equation:

$$
\begin{gathered}
\Phi(x, y, \theta, v, \omega) \\
:=\left[\begin{array}{c}
x+\int_{-r-\tau}^{0} v(s) \cos \left(\theta+\int_{-r-\tau}^{s} \omega(p) d p\right) d s \\
y+\int_{-r-\tau}^{0} v(s) \sin \left(\theta+\int_{-r-\tau}^{s} \omega(p) d p\right) d s \\
\theta+\int_{-r-\tau}^{0} \omega(s) d s
\end{array}\right] .
\end{gathered}
$$

Using any stabilizing feedback from [31], [38] for the nonholonomic integrator (4), the coordinate transformation (53)-(55) and the input transformation (56) and Theorem 2.1, we arrive at the following corollary. 
Corollary 4.1: Assume that $k \in C^{1}\left(\Re^{+} \times \Re^{3} ; \Re^{2}\right)$ with $k(t, 0)=0$ for all $t \geq 0$, is a time periodic uniform stabilizer for (4), i.e., the feedback law $u_{1}(t)=k_{1}\left(t, x_{1}(t), x_{2}(t), x_{3}(t)\right)$, $u_{2}(t)=k_{2}\left(t, x_{1}(t), x_{2}(t), x_{3}(t)\right)$ uniformly, globally stabilizes $0 \in \Re^{3}$ for system (4). Then for every $r, \tau \geq 0, T>0$ the sampled-data dynamic feedback

$$
\begin{aligned}
\dot{z}_{x}(t)= & v(t) \cos \left(z_{\theta}(t)\right) \\
\dot{z}_{y}(t)= & v(t) \sin \left(z_{\theta}(t)\right), t \in\left[\tau_{i}, \tau_{i+1}\right) \\
\dot{z}_{\theta}(t)= & \omega(t) \\
z_{x}\left(\tau_{i+1}\right)= & x\left(\tau_{i+1}-r\right)+\int_{\tau_{i+1}-r-\tau}^{\tau_{i+1}} v(s) \\
& \times \cos \left(\theta\left(\tau_{i+1}-r\right)+\int_{\tau_{i+1}-r-\tau}^{s} \omega(p) d p\right) d s \\
z_{y}\left(\tau_{i+1}\right)= & y\left(\tau_{i+1}-r\right) \\
& +\int_{\tau_{i+1}} v(s) \sin \left(\theta\left(\tau_{i+1}-r\right)+\int_{\tau_{i+1}}^{s} \omega(p) d p\right) d s \\
& \tau_{i+1}-r-\tau \\
z_{\theta}\left(\tau_{i+1}\right)= & \theta\left(\tau_{i+1}-r\right)+\int_{\tau_{i+1}-r-\tau}^{\tau_{i+1}-r-\tau} \omega(s) d s
\end{aligned}
$$

where $\tau_{i}=t_{0}+i T, i \in Z^{+}$and

$$
\begin{aligned}
v(t) & =k_{1}\left(t+\tau, X_{1}(t), X_{2}(t), z_{\theta}(t)\right)+X_{2}(t) \omega(t) \\
\omega(t) & =k_{2}\left(t+\tau, X_{1}(t), X_{2}(t), z_{\theta}(t)\right) \\
X_{1}(t) & =z_{x}(t) \cos \left(z_{\theta}(t)\right)+z_{y}(t) \sin \left(z_{\theta}(t)\right) \\
X_{2}(t) & =z_{x}(t) \sin \left(z_{\theta}(t)\right)-z_{y}(t) \cos \left(z_{\theta}(t)\right)
\end{aligned}
$$

achieves uniform global stabilization of $0 \in \Re^{3}$ for system (57).

At this point it should be emphasized that if the smooth, time-varying feedback proposed in [13] for the stabilization of the nonholonomic integrator were used in (61) then the closed-loop system (57) with (59), (60), (61) would be non-uniformly in time globally asymptotically stable (see Remark 2.3 above). Moreover, the reader should compare the result of Corollary 4.1 with the results in [21]: no restrictions for the magnitudes of the delays are imposed in the present work. Fig. 4 shows the evolution of the state variables $x(t)$, $y(t), \theta(t)$ for the closed-loop system (57), (59), (60), (61) with $\tau=r=1 / 2$, initial conditions $t_{0}=0, x(s)=0.2$, $y(s)=1, \theta(s)=-2$ for $s \in[-(1 / 2), 0], v(s)=\omega(s)=1$ for $s \in[-1,0)$ and $k_{1}(t, x)=-x_{1}+x_{2}(\sin (t)-\cos (t))$, $k_{2}(t, x)=-x_{1} x_{2}\left(1+\cos ^{2}(t)\right)-x_{3}-x_{1}^{2} \cos (t)$. The state variables converge to zero as expected. The nominal feedback law $k_{1}(t, x)=-x_{1}+x_{2}(\sin (t)-\cos (t))$, $k_{2}(t, x)=-x_{1} x_{2}\left(1+\cos ^{2}(t)\right)-x_{3}-x_{1}^{2} \cos (t)$ was proposed in [38] for the global uniform stabilization of (4).

If the aim is to design dead-beat controllers, then a different procedure will be applied. The reader should notice that for every sampling period $T>0$ the discontinuous feedback stabilizer

$$
\begin{aligned}
& k_{1}(x)=-\frac{2}{T} \operatorname{sgn}\left(x_{2}\right)\left|x_{2}\right|^{1 / 2}-\frac{2}{T} x_{1} \\
& k_{2}(x)=\frac{1}{T}\left|x_{2}\right|^{1 / 2}
\end{aligned}
$$

for $x \in C_{1}:=\left\{\xi \in \Re^{3}: \xi_{2}\left(2 \xi_{2}-\xi_{1} \xi_{3}\right) \neq 0\right\}$

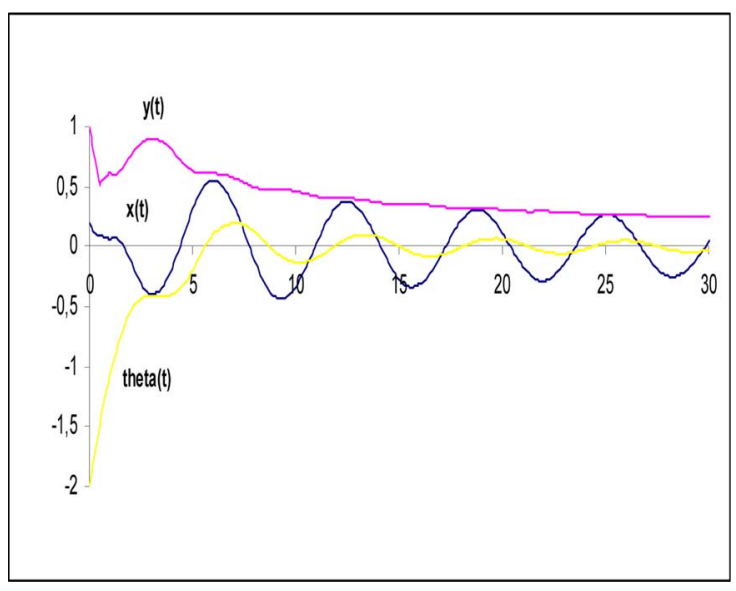

Fig. 4. Evolution of the state variables $x(t), y(t), \theta(t)$ for the closed-loop system (57), (59), and (60), (61) with $\tau=r=0.5$, initial conditions $t_{0}=0$, $x(s)=0.2, y(s)=1, \theta(s)=-2$ for $s \in[-0.5,0], v(s)=\omega(s)=1$ for $s \in[-1,0)$.

$$
\begin{aligned}
k_{1}(x) & =-\frac{x_{1} x_{3}^{2}}{T\left(x_{1}^{2}+x_{3}^{2}\right)}, \quad k_{2}(x)=\frac{x_{3} x_{1}^{2}}{T\left(x_{1}^{2}+x_{3}^{2}\right)} \\
\text { for } x \in C_{2} & :=\left\{\xi \in \Re^{3}: \xi_{2}=0, \xi_{1} \xi_{3} \neq 0\right\} \\
k_{1}(x) & =-\frac{x_{1}}{T} \quad, \quad k_{2}(x)=-\frac{x_{3}}{T} \\
\text { for } x \in C_{3} & :=\left\{\xi \in \Re^{3}: 2 \xi_{2}=\xi_{1} \xi_{3}\right\}
\end{aligned}
$$

satisfies hypothesis (H3) for system (4). To see this notice that inequality (25) holds with $g(s):=2 / T s+3 / T \sqrt{s}$. Furthermore, by explicit computation of the solution one can show that:

- if $x_{0} \in C_{1}$ then the solution $x(t)$ of (4) with $u(t)=k\left(x_{0}\right)$ satisfies $x_{2}(T)=0$, i.e., $x(T) \in C_{2} \cup C_{3}$;

- if $x_{0} \in C_{2}$ then the solution $x(t)$ of (4) with $u(t)=k\left(x_{0}\right)$ satisfies $2 x_{2}(T)=x_{1}(T) x_{3}(T)$, i.e., $x(T) \in C_{3}$;

- if $x_{0} \in C_{3}$ then the solution $x(t)$ of (4) with $u(t)=k\left(x_{0}\right)$ satisfies $x(T)=0$.

It follows that the sampled-data implementation of the feedback (62)-(64) with sampling period $T>0$ guarantees the dead-beat property of order $3 T$ for the corresponding closedloop system. The inequality

$$
\begin{aligned}
\sup _{0 \leq t<T}|x(t)| \leq 3|x(0)|+T(2+|x(0)|) & \sup _{0 \leq t<T}|u(t)| \\
& +\frac{T^{2}}{2} \sup _{0 \leq t<T}|u(t)|^{2}
\end{aligned}
$$

which holds for the solution of (4) for every initial condition and for every applied input $u \in L^{\infty}\left([0, T] ; \Re^{2}\right)$, in conjunction with inequality (25) guarantees for every $T>0$ the existence of a function $a_{T} \in K_{\infty}$ such that for every initial condition the solution of (4) with the sampled-data implementation of the feedback (62)-(65) with sampling period $T>0$ satisfies $\sup _{0<t<3 T}|x(t)| \leq a_{T}(|x(0)|)$. The dead-beat property of order $3 T$ in conjunction with the previous estimate guarantees that the discontinuous feedback stabilizer defined by (62)-(64) satisfies hypothesis (H3) for system (4). The reader should notice that the feedback design procedure described in [8] can be applied as well for the nonholonomic integrator (4) (since (4) is an asymptotically controllable homogeneous system with positive minimal power and zero degree). 
Therefore, we are in a position to solve the finite-time stabilization problem for (57) with measurements $x(t-r), y(t-r)$ and $\theta(t-r)$ available at discrete time instants. We can apply Theorem 3.2 with sampling period $T>0$ for the discontinuous feedback defined by (62)-(64) and obtain the following result.

Proposition 4.2: Let $T>0, r, \tau \geq 0$ with $r+\tau>0$ be given and let $l \in Z^{+}$such that $\tau+r=l T+\delta$ with $0 \leq \delta<T$. Then is uniformly globally asymptotically stable for the closed-loop system (57) with

$$
\begin{aligned}
u(t)= & u_{i}=k_{1}(X \cos (\Theta)+Y \sin (\Theta), X \sin (\Theta) \\
& -Y \cos (\Theta), \Theta), \text { for } t \in\left[\tau_{i}, \tau_{i+1}\right) \\
\omega(t)= & \omega_{i}=k_{2}(X \cos (\Theta)+Y \sin (\Theta), X \sin (\Theta) \\
& -Y \cos (\Theta), \Theta), \text { for } t \in\left[\tau_{i}, \tau_{i+1}\right) \\
v(t)= & u_{i}+\left(t-\tau_{i}\right) \omega_{i}^{2}(X \cos (\Theta)+Y \sin (\Theta)) \\
& +\omega_{i}(X \sin (\Theta)-Y \cos (\Theta)) \\
& +\frac{\left(t-\tau_{i}\right)^{2}}{2} \omega_{i}^{2} u_{i} \text { for } t \in\left[\tau_{i}, \tau_{i+1}\right)
\end{aligned}
$$

where $\tau_{i}=i T, i \in Z^{+}, k: \Re^{3} \rightarrow \Re^{2}$ is defined by (62)-(64) and

$$
\begin{aligned}
X= & x\left(\tau_{i}-r\right) \\
& +\int_{\tau_{i}-r-\tau}^{\tau_{i}} v(s) \cos \left(\theta\left(\tau_{i}-r\right)+\int_{\tau_{i}-r-\tau}^{s} \omega(p) d p\right) d s \\
Y= & y\left(\tau_{i}-r\right) \\
& +\int_{\tau_{i}-r-\tau}^{\tau_{i}} v(s) \sin \left(\theta\left(\tau_{i}-r\right)+\int_{\tau_{i}-r-\tau}^{s} \omega(p) d p\right) d s \\
\Theta= & \theta\left(\tau_{i}-r\right)+\int_{\tau_{i}-r-\tau}^{\tau_{i}} \omega(s) d s .
\end{aligned}
$$

Moreover, for every initial condition the solution of the closedloop system (57) with (65)-(67), (68) where $\tau_{i}=i T, i \in Z^{+}$, $k: \Re^{3} \rightarrow \Re^{2}$ is defined by (62)-(64) satisfies $x(t)=y(t)=$ $\theta(t)=0$ for all $t \geq(l+5) T$.

Although Theorem 3.2 allows the computation of a control action which is applied to (4) with zero order hold, it should be noticed that the control action computed by (65)-(67), (68) is not applied with zero-order hold, because $v(t)$ is not constant on each interval $\left[\tau_{i}, \tau_{i+1}\right), i \in Z^{+}$. This happens because the input transformation (56) is state-dependent.

\section{CONCLUding REMARKS}

Stabilization is studied for nonlinear systems with input and measurement delays, and with measurements available only at discrete time instants (sampling times). Two different cases are considered: the case where the input can be continuously adjusted and the case where the input is applied with zero-order hold. Under the assumption of forward completeness and certain additional stabilizability assumptions, it is shown that sampled-data feedback laws with a predictor-based delay compensation can guarantee global asymptotic stability for the closed- loop system with no restrictions for the magnitude of the delays and arbitrarily long sampling period. Applications to the stabilization of linear networked control systems, strict feedforward systems and a nonholonomic mobile robot over a long-distance communication network are presented.

Solving the ODE (9) as well as finding the value of the predictor map in real time requires care and it may involve a tradeoff between accuracy and calculation time. For the unicycle, a simple Euler scheme is adequate for solving (59).

Future work will address the issue of robustness of the proposed feedback laws with respect to actuator and measurement errors, as well as the extension of the obtained results to the case where the delayed and sampled measured output does not necessarily coincide with the state vector. A different possible future line of research could be the extension of the results in [3]-[5], [26] and the study of the quantization effect.

\section{APPENDIX \\ PROOFS OF CLAIMS}

Proof of Claim 1 in the Proof of Theorem 2.1: Let $\left(x_{0}, z_{0}, u_{0}\right) \in C^{0}\left([-r, 0] ; \Re^{n}\right) \times \Re^{n} \times L^{\infty}\left([-r-\tau, 0] ; \Re^{m}\right)$ be arbitrary and consider the solution $(x(t), z(t), u(t)) \in$ $\Re^{n} \times \Re^{n} \times \Re^{m}$ of the closed-loop system (9), (10), (3), (1) with initial condition $z\left(t_{0}\right)=z_{0} \in \Re^{n}, T_{r+\tau}\left(t_{0}\right) u=u_{0} \in$ $L^{\infty}\left([-r-\tau, 0] ; \Re \Re^{m}\right), T_{r}\left(t_{0}\right) x=x_{0} \in C^{0}\left([-r, 0] ; \Re^{n}\right)$. It is crucial to notice that the solution of $\dot{z}(t)=f(z(t), k(t+$ $\tau, z(t)))$ with $z\left(t_{0}\right)=z_{0} \in \Re^{n}$ satisfies $z(t)=\xi(t+\tau)$, where $\xi(s)$ is the solution of $\dot{\xi}(s)=f(\xi(s), k(s, \xi(s)))$ with $\xi\left(t_{0}+\tau\right)=z_{0} \in \Re^{n}$. Inequality (8) implies that the solution $z(t) \in \Re^{n}$ exists for all $t \in\left[t_{0}, t_{0}+T\right)$ and that the following inequality holds:

$$
|z(t)| \leq \sigma\left(\left|z_{0}\right|, t-t_{0}\right) \forall t \in\left[t_{0}, t_{0}+T\right) .
$$

It follows that the solution of (10) exists for all $t \in$ $\left[t_{0}, t_{0}+T\right)$. Continuity of $k \in C^{1}\left(\Re^{+} \times \Re^{n} ; \Re^{m}\right)$ and inequalities (7), (69) imply that the mapping $t \rightarrow u(t)$ is continuous on $\left(t_{0}, t_{0}+T\right)$ and bounded with $\lim _{t \rightarrow t_{0}^{+}} u(t)=$ $k\left(t_{0}+\tau, z_{0}\right)$ and $\lim _{t \rightarrow\left(t_{0}+T\right)}-u(t)=k\left(t_{0}+T+\tau, z^{*}\right)$,

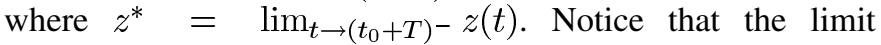
$z^{*}=\lim _{t \rightarrow\left(t_{0}+T\right)^{-}} z(t)$ exists by virtue of uniform continuity of the mapping $t \rightarrow z(t)$ on $\left[t_{0}, t_{0}+T\right)$. By virtue of inequalities (7) and (69) we obtain the inequality

$$
\sup _{t_{0}-\tau-r \leq s<t_{0}+T}|u(s)| \leq\left\|T_{r+\tau}\left(t_{0}\right) u\right\|_{r+\tau}+g\left(\sigma\left(\left|z_{0}\right|, 0\right)\right) .
$$

Using hypothesis (H1) we may conclude that the solution $x(t) \in \Re^{n}$ of (1) exists for all $t \in\left[t_{0}, t_{0}+T\right]$. Indeed, by virtue of the fact, we can guarantee the existence of $\zeta \in K_{\infty}$ such that

$|x(t)| \leq \zeta\left(\left|x\left(t_{0}\right)\right|+\sup _{t_{0}-\tau \leq s<t-\tau}|u(s)|\right)$, for all $t \in\left(t_{0}, t_{0}+T\right]$.

The above inequality in conjunction with (70) and the trivial inequality $\left\|T_{r}(t) x\right\|_{r} \leq\left\|T_{r}\left(t_{0}\right) x\right\|_{r}+\sup _{t_{0} \leq s \leq t}|x(s)|$ gives

$$
\begin{aligned}
\left\|T_{r}(t) x\right\|_{r} \leq\left\|x_{0}\right\|_{r}+ & \zeta\left(\left\|x_{0}\right\|_{r}+\left\|T_{r+\tau}\left(t_{0}\right) u\right\|_{r+\tau}\right. \\
& \left.+g\left(\sigma\left(\left|z_{0}\right|, 0\right)\right)\right) \forall t \in\left[t_{0}, t_{0}+T\right] .
\end{aligned}
$$


Finally, we define $u\left(t_{0}+T\right)=k\left(t_{0}+T+\tau, z\left(t_{0}+T\right)\right)$, where $z\left(t_{0}+T\right)=\Phi\left(x\left(t_{0}+T-r\right), T_{r+\tau}\left(t_{0}+T\right) u\right)$. Again, using (5), (7), (70) and (71) we obtain

$$
\begin{aligned}
\left|z\left(t_{0}+T\right)\right| \leq & a\left(\left\|T_{r+\tau}\left(t_{0}\right) u\right\|_{r+\tau}\right. \\
& \left.+g\left(\sigma\left(\left|z_{0}\right|, 0\right)\right)+\left\|x_{0}\right\|_{r}+M\right) \\
\left|u\left(t_{0}+T\right)\right| \leq & g\left(a \left(\left\|T_{r+\tau}\left(t_{0}\right) u\right\|_{r+\tau}\right.\right. \\
& \left.\left.+g\left(\sigma\left(\left|z_{0}\right|, 0\right)\right)+\left\|x_{0}\right\|_{r}+M\right)\right)
\end{aligned}
$$

where $M=\zeta\left(\left\|x_{0}\right\|_{r}+\left\|T_{r+\tau}\left(t_{0}\right) u\right\|_{r+\tau}+g\left(\sigma\left(\left|z_{0}\right|, 0\right)\right)\right)$. Using (69), (70), (71), and the above inequalities we are in a position to construct a function $G \in K_{\infty}$ such that inequality (12) holds. The proof is complete. $\triangleleft$

Proof of Claim 3 in the Proof of Theorem 3.2: The claim is proved by induction on $p \in Z^{+}, p \geq 1$. First we show that the claim holds for $p=1$. Let arbitrary $\left(x_{0}, u_{0}\right) \in C^{0}\left([-r, 0] ; \Re^{n}\right) \times L^{\infty}\left([-r-\tau, 0) ; \Re^{m}\right)$, and consider the solution $(x(t), u(t)) \in \Re^{n} \times \Re^{m}$ of system (28) with initial condition $\breve{T}_{r+\tau}(0) u=u_{0} \in L^{\infty}\left([-r-\tau, 0) ; \Re^{m}\right)$, $T_{r}(0) x=x_{0} \in C^{0}\left([-r, 0] ; \Re^{n}\right)$. Using the equation $u(t)=k\left(\Phi\left(x(-r), \breve{T}_{r+\tau}(0) u\right)\right), t \in[0, T)$, in conjunction with inequalities (5) and (25), we obtain

$$
\sup _{0 \leq s<T}|u(s)| \leq g\left(a\left(|x(-r)|+\left\|u_{0}\right\|_{r+\tau}\right)\right) .
$$

Inequality (72) implies the following inequality:

$$
\begin{aligned}
\sup _{-\tau-r \leq s<T}|u(s)| \leq & \left\|u_{0}\right\|_{r+\tau}+\sup _{0 \leq s<T}|u(s)| \\
\leq & \left\|u_{0}\right\|_{r+\tau} \\
& +g\left(a\left(\left\|x_{0}\right\|_{r}+\left\|u_{0}\right\|_{r+\tau}\right)\right) .
\end{aligned}
$$

Exploiting (30) and (73) in conjunction with the trivial inequalities $|x(0)| \leq\left\|x_{0}\right\|_{r}$ and $\sup _{-r \leq s \leq T}|x(s)| \leq\left\|x_{0}\right\|_{r}+$ $\sup _{0<s \leq T}|x(s)|$, we obtain

$$
\sup _{-r \leq t \leq T}|x(t)| \leq\left\|x_{0}\right\|_{r}+b(M+g(a(M)))
$$

where $M=\left\|x_{0}\right\|_{r}+\left\|u_{0}\right\|_{r+\tau}$. Inequalities (73) and (74) directly imply that (31) holds with $G_{1}(s):=s+g(a(s))+$ $b(s+g(a(s)))$. Thus, the claim holds for $p=1$.

Next, we suppose that the claim holds for certain $p \in Z^{+}$, $p \geq 1$. We will show that the claim holds for $p+1$. Let arbitrary $\left(x_{0}, u_{0}\right) \in C^{0}\left([-r, 0] ; \Re^{n}\right) \times L^{\infty}\left([-r-\tau, 0) ; \Re^{m}\right)$, and consider the solution $(x(t), u(t)) \in \Re^{n} \times \Re^{m}$ of system (28) with initial condition $\widetilde{T}_{r+\tau}(0) u=u_{0} \in L^{\infty}\left([-r-\tau, 0) ; \Re^{m}\right)$, $T_{r}(0) x=x_{0} \in C^{0}\left([-r, 0] ; \Re^{n}\right)$. There exists a function $G_{p} \in K_{\infty}$ such that (31) holds. Using the equation
$u(t)=k\left(\Phi\left(x(p T-r), \breve{T}_{r+\tau}(p T) u\right)\right), t \in[p T,(p+1) T)$, in conjunction with inequalities (5), (25) and (31), we obtain

$$
\begin{aligned}
\sup _{p T \leq s<(p+1) T}|u(s)| & \leq g\left(a\left(|x(p T-r)|+\sup _{-r-\tau \leq s<p T}|u(s)|\right)\right) \\
& \leq g\left(a\left(G_{p}\left(\left\|x_{0}\right\|_{r}+\left\|u_{0}\right\|_{r+\tau}\right)\right)\right) .
\end{aligned}
$$

Inequality (75) implies the following inequality:

$$
\begin{aligned}
\sup _{-\tau-r \leq s<(p+1) T}|u(s)| \leq & \sup _{-\tau-r \leq s<p T}|u(s)| \\
& +\sup _{p T \leq s<(p+1) T}|u(s)| \\
\leq & \sup _{-\tau-r \leq s<p T}|u(s)| \\
& +g\left(a\left(G_{p}\left(\left\|x_{0}\right\|_{r}+\left\|u_{0}\right\|_{r+\tau}\right)\right)\right) .
\end{aligned}
$$

On the other hand, by exploiting (30) (which gives $|x(t)| \leq b\left(|x(p T)|+\sup _{p T-\tau \leq s<t-\tau}|u(s)|\right)$ for all $t \in$ $(p T,(p+1) T])$ and (76) in conjunction with the trivial inequalities $|x(p T)| \leq \sup _{-r \leq s \leq p T}|x(s)|, \sup _{-r \leq s \leq(p+1) T}|x(s)| \leq$ $\sup _{-r \leq s \leq p T}|x(s)|+\sup _{p T<s \leq(p+1) T}|x(s)|$, we obtain

$$
\begin{aligned}
\sup _{-r \leq t \leq(p+1) T} \mid & x(t)\left|\leq \sup _{-r \leq t \leq p T}\right| x(t) \mid \\
& +b\left(M+g\left(a\left(G_{p}\left(\left\|x_{0}\right\|_{r}+\left\|u_{0}\right\|_{r+\tau}\right)\right)\right)\right)
\end{aligned}
$$

where $M=\sup _{-r \leq t \leq p T}|x(t)|+\sup _{-r-\tau<t<p T}|u(t)|$. Inequalities (76), (77) in conjunction with (31) directly imply that inequality (31) holds with $p \in Z^{+}$replaced by $p+1$ and $G_{p} \in K_{\infty}$ replaced by $G_{p+1}(s):=G_{1}\left(G_{p}(s)\right)$.

The proof is complete $\triangleleft$.

\section{ACKNOWLEDGMENT}

The authors would like to thank Prof. C. Kravaris for bringing to their attention the fact that the predictor mapping can be explicitly computed for bilinear systems $\dot{x}=A x+B u+u C x$, with $x \in \Re^{n}, u \in \Re$, and $A C=C A$ [see Remark 2.2(d)]. Moreover, the authors would like to thank the anonymous reviewers for their suggestions and comments.

\section{REFERENCES}

[1] D. Angeli and E. D. Sontag, "Forward completeness, unbounded observability and their lyapunov characterizations," Syst. Control Lett., vol. 38, no. 4-5, pp. 209-217, 1999.

[2] B. Castillo-Toledo, S. Di Gennaro, and G. S. Castro, "Stability analysis for a class of sampled nonlinear systems with time-delay," in Proc. 49th Conf. Decision Control, Atlanta, GA, 2010, pp. 1575-1580.

[3] C. De Persis and A. Isidori, "Stabilizability by state feedback implies stabilizability by encoded state feedback," Syst. Control Lett., vol. 53, pp. 249-258, 2004.

[4] C. De Persis, "n-bit stabilization of n-Dimensional nonlinear systems in feedforward form," IEEE Trans. Autom. Control, vol. 50, no. 3, pp. 285-297, Mar. 2005.

[5] C. De Persis, "Minimal data-rate stabilization of nonlinear systems over networks with large delays," Int. J. Robust Nonlinear Control, vol 20, no. 10, pp. 1097-1111, 2010.

[6] E. Fridman, A. Seuret, and J.-P. Richard, "Robust sampled-data stabilization of linear systems: An input delay approach," Automatica, vol. 40, pp. 1441-1446, 2004. 
[7] H. Gao, T. Chen, and J. Lam, "A new system approach to networkbased control," Automatica, vol. 44, no. 1, pp. 39-52, 2008.

[8] L. Grüne, "Homogeneous state feedback stabilization of homogenous systems," SIAM J. Control Optim., vol. 38, no. 4, pp. 1288-1308, 2000.

[9] L. Grüne and D. Nešić, "Optimization based stabilization of sampleddata nonlinear systems via their approximate discrete-time models," SIAM J. Control Optim., vol. 42, pp. 98-122, 2003.

[10] M. Heemels, A. R. Teel, N. van de Wouw, and D. Nešić, "Networked control systems with communication constraints: Tradeoffs between transmission intervals, delays and performance," IEEE Trans. Autom. Control, vol. 55, no. 8, pp. 1781-1796, Aug. 2010.

[11] G. Herrmann, S. K. Spurgeon, and C. Edwards, "Discretization of sliding mode based control schemes," in Proc. 38th Conf. Decision Control, Phoenix, AZ, 1999, pp. 4257-4262.

[12] M. Jankovic, "Recursive predictor design for state and output feedback controllers for linear time delay systems," Automatica, vol. 46, pp. 510-517, 2010.

[13] I. Karafyllis and J. Tsinias, "Global stabilization and asymptotic tracking for a class of nonlinear systems by means of time-varying feedback," Int. J. Robust Nonlinear Control, vol. 13, no. 6, pp. $559-588,2003$.

[14] I. Karafyllis, "A system-theoretic framework for a wide class of systems I: Applications to numerical analysis," J. Math. Anal. Applicat., vol. 328, no. 2, pp. 876-899, 2007.

[15] I. Karafyllis and Z.-P. Jiang, "A small-gain theorem for a wide class of feedback systems with control applications," SIAM J. Control Optim., vol. 46, no. 4, pp. 1483-1517, 2007.

[16] I. Karafyllis and C. Kravaris, "Global stability results for systems under sampled-data control," Int. J. Robust Nonlinear Control, vol. 19, no. 10, pp. $1105-1128,2009$

[17] I. Karafyllis, P. Pepe, and Z.-P. Jiang, "Stability results for systems described by coupled retarded functional differential equations and functional difference equations," Nonlinear Anal., Theory, Meth., Applicat. vol. 71, no. 7-8, pp. 3339-3362, 2009.

[18] I. Karafyllis and C. Kravaris, "Robust global stabilizability by means of sampled-data control with positive sampling rate," Int. J. Control, vol. 82, no. 4, pp. 755-772, 2009.

[19] I. Karafyllis, "Stabilization by means of approximate predictors for systems with delayed input," SIAM J. Control Optim., vol. 49, no. 3, pp. $1100-1123,2011$.

[20] H. K. Khalil, Nonlinear Systems, 2nd ed. Englewood Cliffs, NJ: Prentice-Hall, 1996.

[21] K. Kojima, T. Oguchi, A. Alvarez-Aguirre, and H. Nijmeijer, "Predictor-Based tracking control of a mobile robot with time-delays," in Proc. NOLCOS '10, Bologna, Italy, 2010, pp. 167-172.

[22] M. Krstic, "Feedback linearizability and explicit integrator forwarding controllers for classes of feedforward systems," IEEE Trans. Autom. Control, vol. 49, no. 10, pp. 1668-1682, Oct. 2004.

[23] M. Krstic, Delay Compensation for Nonlinear, Adaptive, and PDE Systems. Systems \& Control: Foundations \& Applications. Boston, MA: Birkhäuser, 2009.

[24] M. Krstic, "Input delay compensation for forward complete and feedforward nonlinear systems," IEEE Trans. Autom. Control, vol. 55, no. 2, pp. 287-303, Feb. 2010.

[25] M. Krstic, "Lyapunov stability of linear predictor feedback for timevarying input delay," IEEE Trans. Autom. Control, vol. 55, no. 2, pp. 554-559, Feb. 2010.

[26] D. Liberzon and J. P. Hespanha, "Stabilization of nonlinear systems with limited information feedback," IEEE Trans. Autom. Control, vol. 50, no. 6, pp. 910-915, Jun. 2005.

[27] R. Lozano, P. Castillo, P. Garcia, and A. Dzul, "Robust predictionbased control for unstable delay systems: Application to the yaw control of a mini-helicopter," Automatica, vol. 40, pp. 603-612, 2004.

[28] F. Mazenc, S. Mondie, and R. Francisco, "Global asymptotic stabilization of feedforward systems with delay at the input," IEEE Trans. Autom. Control, vol. 49, no. 5, pp. 844-850, May 2004.

[29] F. Mazenc and P.-A. Bliman, "Backstepping design for time-delay nonlinear systems," IEEE Trans. Autom. Control, vol. 51, no. 1, pp. 149-154, Jan. 2006.

[30] F. Mazenc, M. Malisoff, and Z. Lin, "Further results on input-to-state stability for nonlinear systems with delayed feedbacks," Automatica, vol. 44, pp. 2415-2421, 2008.

[31] P. Morin, J.-B. Pomet, and C. Samson, "Design of homogeneous timevarying stabilizing control laws for driftless controllable systems via oscillatory approximation of lie brackets in closed loop," SIAM J. Control Optim., vol. 38, no. 1, pp. 22-49, 1999.

[32] P. Naghshtabrizi, J. Hespanha, and A. R. Teel, "Stability of delay impulsive systems with application to networked control systems," in Proc. 26th Amer. Control Conf., New York, 2007, pp. 4899-4904.
[33] D. Nešic, A. R. Teel, and P. V. Kokotovic, "Sufficient conditions for stabilization of sampled-data nonlinear systems via discrete-time approximations," Syst. Control Lett., vol. 38, no. 4-5, pp. 259-270, 1999.

[34] D. Nešic and A. R. Teel, "Sampled-data control of nonlinear systems: An overview of recent results," in Perspectives on Robust Control, R. S. O. Moheimani, Ed. New York: Springer-Verlag, 2001, pp. 221-239.

[35] D. Nešic and A. Teel, "A framework for stabilization of nonlinear sampled-data systems based on their approximate discrete-time models," IEEE Trans. Autom. Control, vol. 49, no. 7, pp. 1103-1122, Jul. 2004.

[36] D. Nešic and D. Liberzon, "A unified framework for design and analysis of networked and quantized control systems," IEEE Trans. Autom. Control, vol. 54, no. 4, pp. 732-747, Apr. 2009.

[37] D. Nešic, A. R. Teel, and D. Carnevale, "Explicit computation of the sampling period in emulation of controllers for nonlinear sampled-data systems," IEEE Trans. Autom. Control, vol. 54, no. 3, pp. 619-624, May 2009.

[38] J.-P. Pomet, "Explicit design of time-varying stabilizing control laws for a class of controllable systems without drift," Syst. Control Lett., vol. 18 , no. 2, pp. 147-158, 1992.

[39] R. Sepulchre, M. Jankovic, and P. Kokotovic, Constructive Nonlinear Control. New York: Springer, 1997.

[40] M. Tabbara, D. Nešic, and A. R. Teel, "Networked control systems: Emulation based design," in Networked Control Systems, ser. Series in Intelligent Control and Intelligent Automation, D. Liu and F.-Y. Wang, Eds. Singapore: World Scientific, 2007.

[41] P. Tabuada, "Event-Triggered real-time scheduling of stabilizing control tasks," IEEE Trans. Autom. Control, vol. 52, no. 9, pp. 1680-1685, Sep. 2007.

[42] A. R. Teel, "Connections between Razumikhin-type theorems and the ISS nonlinear small gain theorem," IEEE Trans. Autom. Control, vol. 43, no. 7, pp. 960-964, Jul. 1998.

[43] M. Yu, L. Wang, T. Chu, and F. Hao, "Stabilization of networked control systems with data packet dropout and transmissions delays: Continuous-time case," Eur. J. Control, vol. 11, no. 1, pp. 41-49, 2005.

[44] X. Zhang, H. Gao, and C. Zhang, "Global asymptotic stabilization of feedforward nonlinear systems with a delay in the input," Int. J. Syst. Sci., vol. 37, no. 3, pp. 141-148, 2006.

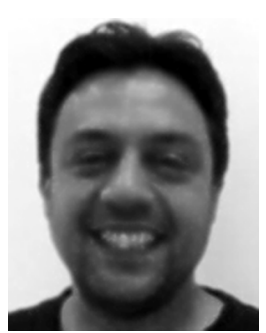

Iasson Karafyllis received the B.S. degree in chemical engineering from the National Technical University of Athens (NTUA), Athens, Greece, in 1994, the M.Sc. degree in mathematics from the University of Minnesota, Minneapolis, in 1997, and the Ph.D. degree in mathematics from the NTUA, in 2003.

$\mathrm{He}$ is currently an Assistant Professor in the Department of Environmental Engineering at the Technical University of Crete, Chania, Greece. His research interests include mathematical systems and control theory, stability theory, and robust feedback stabilization problems for deterministic systems. He is a coauthor of the book Stability and Stabilization of Nonlinear Systems (Springer, 2011).

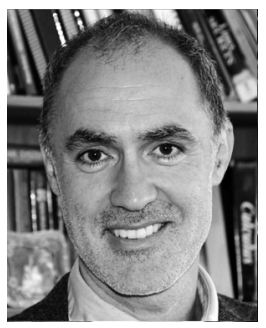

Miroslav Krstic (S'92-M'95-SM'99-F'02) received the Ph.D. degree from the University of California (UC), Santa Barbara, in 1994.

$\mathrm{He}$ is the Daniel L. Alspach Professor and the founding Director of the Cymer Center for Control Systems and Dynamics (CCSD) at UC San Diego, La Jolla. He was an Assistant Professor at University of Maryland until 1997. He is a coauthor of eight books: Nonlinear and Adaptive Control Design (Wiley, 1995), Stabilization of Nonlinear Uncertain Systems (Springer, 1998), Flow Control by Feedback (Springer, 2002), Real-time Optimization by Extremum Seeking Control (Wiley, 2003), Control of Turbulent and Magnetohydrodynamic Channel Flows (Birkhauser, 2007), Boundary Control of PDEs: A Course on Backstepping Designs (SIAM, 2008), Delay Compensation for Nonlinear, Adaptive, and PDE Systems (Birkhauser, 2009), and Adaptive Control of Parabolic PDEs (Princeton, 2010).

Prof. Krstic is a Fellow of IFAC and has received the Axelby and Schuck paper prizes, NSF Career, ONR Young Investigator, and PECASE awards. He has held the appointment of Springer Distinguished Visiting Professor of Mechanical Engineering at UC Berkeley. 